\title{
Atomic-scale microstructure of metal halide perovskite
}

Authors: Mathias Uller Rothmann ${ }^{1}$, Judy S. Kim ${ }^{2,3,4}$, Juliane Borchert ${ }^{1}$, Kilian Lohmann ${ }^{1}$, Colum M. O’Leary ${ }^{2}$, Alex Sheader ${ }^{2}$, Laura Clark ${ }^{2}$, Henry J. Snaith ${ }^{1}$, Michael B. Johnston ${ }^{1}$, Peter D. Nellist ${ }^{2}$, and Laura M. Herz ${ }^{1 *}$

\author{
Affiliations: \\ ${ }^{1}$ Department of Physics, University of Oxford, Clarendon Laboratory, Parks Road, Oxford \\ OX1 3PU, UK \\ ${ }^{2}$ Department of Materials, University of Oxford, Parks Road, Oxford OX1 3PH, UK \\ ${ }^{3}$ ePSIC, Diamond Light Source, Harwell, Didcot, OX11 0DE, UK \\ ${ }^{4}$ Rosalind Franklin Institute, Fermi Ave, Harwell, Didcot, OX11 0QS, UK \\ *Correspondence to: laura.herz@physics.ox.ac.uk
}

\section{One Sentence Summary:}

The atomic-scale microstructure of metal halide perovskites is revealed by high-resolution, low-dose electron microscopy.

\begin{abstract}
:
Hybrid organic-inorganic perovskites are exciting materials for solar-energy applications whose microscopic properties are still not well understood. Atomic-resolution (scanning) transmission electron microscopy, (S)TEM, has provided invaluable insights for many crystalline solar-cell materials, and is used here to successfully image $\mathrm{CH}\left(\mathrm{NH}_{2}\right)_{2} \mathrm{PbI}_{3}$ thin films with low electron-radiation dose. Such images reveal a highly ordered atomic arrangement of sharp grain boundaries and coherent perovskite/ $\mathrm{PbI}_{2}$ interfaces, with a
\end{abstract}


striking absence of long-range disorder in the crystal. We demonstrate that beaminduced degradation of the perovskite leads to an initial loss of $\mathrm{CH}\left(\mathrm{NH}_{2}\right)_{2}{ }^{+}$ions, leaving behind a partially unoccupied perovskite lattice, which explains the unusual regenerative properties of these materials. We further observe aligned point defects and climbdissociated dislocations. Our findings thus provide an atomic-level understanding of technologically important lead-halide perovskites.

\section{Main Text:}

Many of the phenomenal advances in the performance of hybrid metal halide perovskite (MHP) solar cells have resulted from improvements in device architecture and material composition studied on a mesoscopic to macroscopic scale(1-7), and changes in material properties have usually been inferred from relatively "large area" probes $(8-11)$. While these probes are able to resolve properties on the scale of a few hundred nanometres to a few millimetres, the mechanisms behind the impressive performance of hybrid perovskites have yet to be fully understood, and are bound to link directly with unique properties at the atomic level. This point is vividly illustrated by effects such as the gradual rather than abrupt decrease in photovoltaic performance of MHPs over time(12), their readily regenerative properties when exposed to gaseous iodo-organic precursors(13), and the counterintuitively beneficial effects of adding a small excess $(2 \%(14,15)-7.5 \%(16))$ of $\mathrm{PbI}_{2}$ to the MHP precursor solution. Similarly, significant efforts have been made to describe the properties of grain boundaries in MHP thin films using light and scanning probes(17-19) with highly inconsistent results $(11,17)$. Recent work has shown that a smaller spread of grain boundary orientations increases the photoluminescence intensity locally $(20)$, but the exact atomic nature of grain boundaries has still not been described. Even the crystal phase of the technologically important $\mathrm{CH}\left(\mathrm{NH}_{2}\right)_{2} \mathrm{PbI}_{3}$ (formamidinium lead iodide, $\mathrm{FAPbI}_{3}$ ) $\mathrm{MHP}$ is still uncertain(21). Therefore, truly atomic 
resolution imaging of metal halide perovskites has phenomenal potential to provide urgently needed understanding for a plethora of puzzling observations already established for perovskite devices, and in turn, boost their efficiencies further. In addition, grain boundaries have been postulated to have a significant impact on the long-term stability of $\operatorname{MHPs}(22,23)$, yet their exact nature is still mostly unknown.

These examples illustrate that a detailed understanding of the atomic structure of MHPs is urgently required in order to address these fundamental issues and allow further development of perovskite solar cells and other optoelectronic technologies. Atomic-resolution transmission electron microscopy has uniquely answered similar questions for other crystalline solar cell materials $(24,25)$, but the highly beam-sensitive nature of hybrid perovskites makes highresolution electron microscopy extremely challenging. While fully inorganic photoactive perovskites have been successfully imaged(26), the few studies observing the pristine hybrid perovskite phase are based on nanostructures $(27,28)$, and no studies of pristine, native hybrid perovskite thin films have to date been published. Unstable under electron beams, MHPs rapidly change into a more stable $\mathrm{PbI}_{2}$ phase under even mild electron irradiation $(29,30)$, which has resulted in significant differences in the crystallography reported by electron microscope and X-ray studies(11).

\section{Imaging the perovskite structure}

We employ low-dose scanning transmission electron microscopy to obtain images of pristine $\mathrm{FAPbI}_{3}$, as STEM has been shown for other materials to produce less damage with the same total electron fluence (dose)(31). In order to resolve both "near-stoichiometric" polycrystalline $\mathrm{FAPbI}_{3}$ films, and those formed with significant excess $\mathrm{PbI}_{2}$ precursor (" $\mathrm{PbI}_{2}$-rich"), we prepared two different types of thermally evaporated materials on ultra-thin carbon-coated copper TEM grids: thinner 30-nm $\mathrm{FAPbI}_{3}$ films close to stoichiometry (FA:Pb:I ratios close to 1:1:3, containing a small excess of $\mathrm{PbI}_{2}$ as used to optimize for solar cell applications(32)) and 
thicker 90-nm films containing significant excess $\mathrm{PbI}_{2}$. To examine whether similar atomicscale microstructures were also observable for $\mathrm{MAPbI}_{3}$ we also deposited $\mathrm{MAPbI}_{3}$ films of 30 nm thickness containing significant excess $\mathrm{PbI}_{2}$. Full details of the sample preparation procedures and characterization are provided in the Supplementary Materials and Fig. S1. We have reported thin films of $\mathrm{FAPbI}_{3}$ and $\mathrm{MAPbI}_{3}$ deposited by us from such dual-source vapordeposition protocols to achieve photovoltaic power conversion efficiencies of $14-19 \%$ when incorporated into suitable device $\operatorname{architectures}(23,33)$. STEM samples were annealed according to protocols previously developed to optimize photovoltaic performance. In Fig. 1 we show a low-angle annular dark field (LAADF, inner radius of $33 \mathrm{mrad}$, see Methods for full low-dose image acquisition conditions) STEM micrograph of a 90-nm thick $\mathrm{FAPbI}_{3}$ film containing excess $\mathrm{PbI}_{2}$. LAADF is similar to high-angle annular dark field (HAADF) as it detects scattered electrons, making it sensitive to atomic number variations, but has the advantage of collecting a higher fraction of the scattered electrons, leading to a higher atomic detection efficiency. In addition, LAADF allows efficient imaging of lower- $Z$ elements, which scatter at lower angles(34). In order to improve the contrast of this image of a pristine crystal, we applied a Bragg filter, as described in detail the Methods Section and Fig. S2.
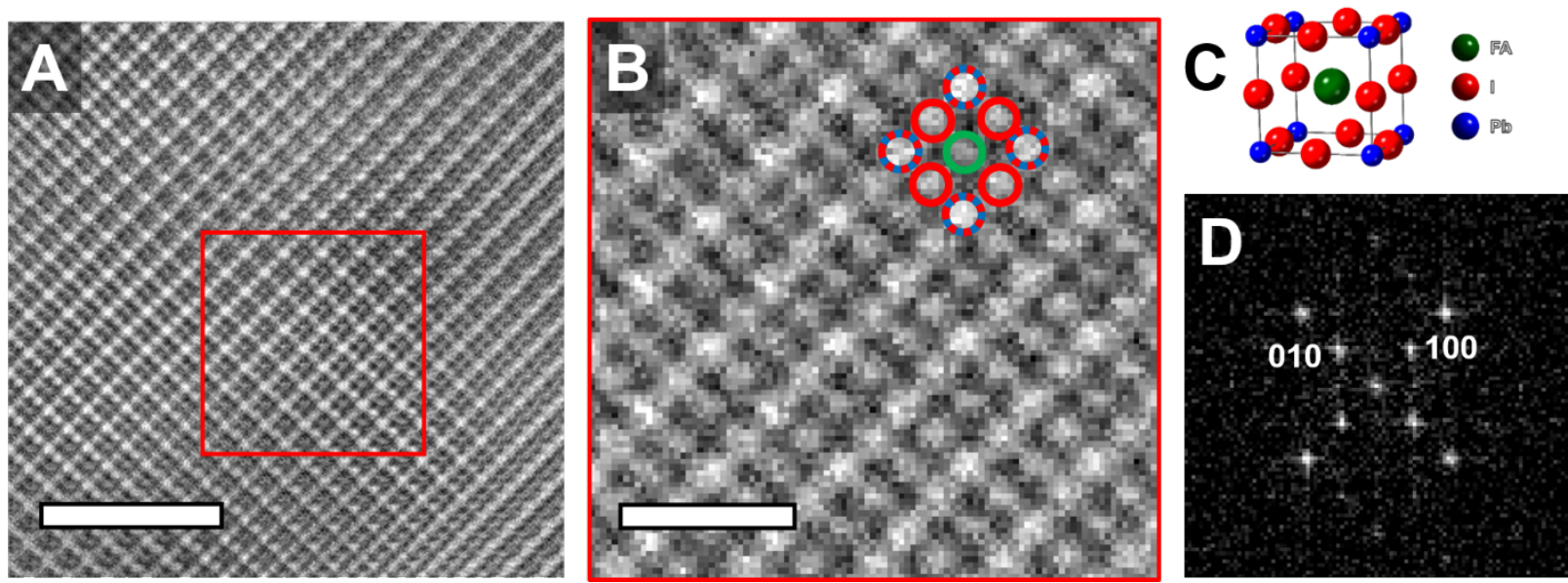

Fig. 1: Atomic resolution imaging of the metal halide perovskite structure. (A) Bragg-filtered atomic resolution $\mathrm{LAADF}$ micrograph of a pristine 90-nm thick, $<001>$-oriented $\mathrm{FAPbI}_{3}$ film fabricated with excess $\mathrm{PbI}_{2}$. A distinct pattern in the contrast of the individual atomic columns 
is clearly visible, with the area in the red square magnified in (B). Here, the variations in composition appear as differences in contrast, the dashed blue and red circles showing the Pb/I columns, the red circles showing the pure I columns, and the green circles showing the $F A^{+}$columns, indicated in the atomic unit cell seen in (C). The Fourier transformation (FT) in (D) confirms the presence of the $\{010\}$ and $\{100\}$ planes, which are often missing from atomicresolution studies of beam-damaged $\mathrm{FAPbI}_{3}$. The scale bars in $(A, B)$ represent 5 and $1 \mathrm{~nm}$, respectively. The total dose used for acquisition of images (A) and (B) is $\sim 100$ e $A^{-2} . A$ comparison of the full image, for raw (unfiltered), Butterworth-and Bragg-filtered data is shown in Fig. S2.

The lower magnification image in Fig. 1A reveals an atomic lattice pattern consistent with a cubic structure observed along the $<001>$ orientation. The magnified image in Fig. 1B clearly shows the atomic-level crystal structure corresponding to the cubic structure of roomtemperature $\mathrm{FAPbI}_{3}$, displayed schematically in Fig. $1 \mathrm{C}(21)$. We note that the preferred alignment of growth along the [100] axis ("face-up") is generally observed by us in these dualsource vapor-deposited films, and is common for lead halide perovskite deposited across a variety of alternative processing techniques(35). We also note that an important advantage of LAADF imaging in this case is that, similarly to HAADF imaging, the column intensities retain a monotonic dependence on atomic number. For completeness, a comparison with a HAADF image, acquired as described in the Methods, is shown in Fig. S3.

Three levels of intensity are observable in the LAADF images: the brightest corresponds to the corners of the cubic unit cell (dashed blue and red circles in Fig. 1B), containing alternating lead and iodine, both strongly scattering elements. The second brightest intensity corresponds to the edges of the unit cell containing only iodine, illustrated by the red circles in Fig. 1B. In the $z$ viewing direction, the corners make up a total of one lead atom and one iodine per unit 
cell, whereas the edge position only contains one iodine, resulting in less scattering and a less bright image intensity. The central, lowest intensity position corresponds to the $\mathrm{FA}^{+}$ions, which scatter the least because of their organic nature and singular presence in the centre of the unit cell(34). Intensity line profiles and a multislice simulation verifying the differences in contrast are provided in Fig. S4.

To support structure identification, we performed a Fourier transformation (FT, shown in Fig. 1D) on the area marked in Fig. 1A, clearly identifying the presence of the (100) and (010) planes along with the (200) and (020) planes. Out of the three proposed room-temperature crystal phases of $\mathrm{FAPbI}_{3}$ (cubic, trigonal, and hexagonal(21)), the spots corresponding to the (100) and (010) planes are unique to the cubic perovskite structure and cannot correspond to either of the other two. This strongly indicates that the room-temperature phase of $\mathrm{FAPbI}_{3}$ is cubic in the $\mathrm{Pm} \overline{3} \mathrm{~m}$ space group.

We note that the (100) and (010) FT spots evident in Fig. 1D have been absent in previous atomic-resolution (S)TEM studies of both $\mathrm{FAPbI}_{3}$ and $\mathrm{MAPbI}_{3}$, with only the supposed (200) and the (020) (plane spacings of $3.181 \AA$ ) or other higher index spots still visible(36-39). Broad-beam diffraction studies carried out on $\mathrm{MAPbI}_{3}$ have explained this apparent absence of lower-order diffraction signatures as the result of structural damage caused by high electron irradiation dosage $(29,30)$. Thus, the spots attributed to the second-order diffraction off the perovskite lattice may instead derive from the $\mathrm{PbI}_{2}$ formed as a decomposition product under intense electron irradiation(40, 41).

\section{Electron-induced decomposition}

Using a suitably chosen irradiation dosage, we are indeed able to observe the gradual decomposition of $\mathrm{FAPbI}_{3}$ with electron beam exposure. Fig. 2A shows a lower magnification Butterworth-filtered LAADF image of a pristine grain boundary in a $30-\mathrm{nm}$ thick $\mathrm{FAPbI}_{3}$ film close to stoichiometry on a carbon-coated TEM grid after a single scan of the electron beam, 
with a total dose of $\sim 66 \mathrm{e} \AA^{-2}$. The subsequent scans were also recorded and are provided in Fig. 2B-D. After three scans $\left(\sim 200 \mathrm{e}^{-2}\right)$, the contrast within the perovskite structure has visibly changed, and after nine scans $\left(\sim 600 \mathrm{e}^{-2}\right)$, the perovskite structure is completely depleted and replaced by a crystal structure with uniform column intensities, displayed in Fig. 2C. Further extensive exposure $\left(\sim 1333 \mathrm{e}^{-2}\right)$ slightly alters this phase into one very similar to $2 \mathrm{H}$ hexagonal $\mathrm{PbI}_{2}$, displayed in Fig. 2D. This final structure is highly stable, does not change significantly after further exposure and corresponds well to the $\mathrm{PbI}_{2}$ phase observed by diffraction in broad beam TEM studies(29). A full crystallographic determination of the phase seen in Fig. 2C is beyond the scope of this study, but Fig. S10 shows how a small distortion of hexagonal $\mathrm{PbI}_{2}$ can form a structure that projects as a square lattice and forms a coherent epitaxial interface with $\mathrm{FAPbI}_{3}$ as observed in the image data. The FTs in Fig. 2E-H show the degradation process on a crystallographic level: for the pristine perovskite, diffraction spots corresponding to the $\{100\}$ plane family are initially clearly visible (Fig. 2E). Additional spots appear after brief exposure (orange circles, Fig. 2F), likely corresponding to the ordered loss of formamidinium ions discussed in full detail further below. Continued exposure fully destroys the perovskite structure, transforming it into electron-irradiation-stable $\mathrm{PbI}_{2}$ concomitant with the disappearance of the $\{100\}$ plane family of the original perovskite (Fig. 2F), and finally into a structure similar to that of $2 \mathrm{H}$-hexagonal $\mathrm{PbI}_{2}$ (Fig. $\left.2 \mathrm{H}\right)$. 

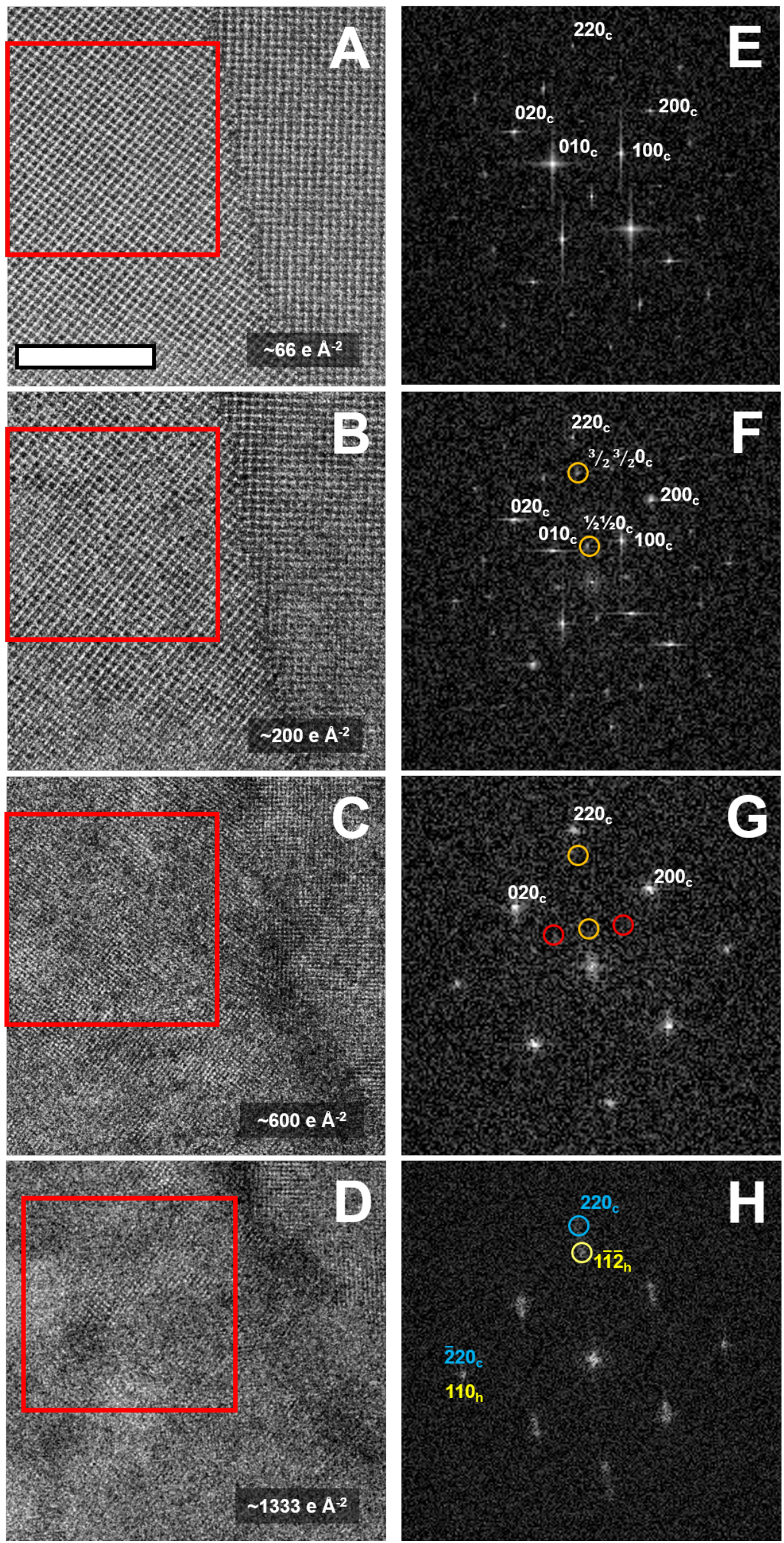
Fig. 2: Damage mechanism of $\mathrm{FAPbI}_{3}$ under a scanning electron beam observed in a 30nm thick film prepared near the correct stoichiometry. (A) The first scan $\left(\sim 66 e^{-2}\right)$ shows the pristine perovskite structure and a grain boundary. (B) After three scans ( 200 e $\left.\AA^{-2}\right)$, changes in the crystal structure are apparent in the LAADF image but the grain boundary is still distinguishable. (C) After nine scans $\left(\sim 600 \mathrm{e}^{-2}\right)$ the perovskite structure has fully disappeared and a new crystalline phase has replaced it. Different crystal orientations are visible, but there is no clear grain boundary. (D) After 20 scans ( 1333 $\left.e A^{-2}\right)$, the angles of the crystal plans change slightly, indicating the complete transformation into $2 \mathrm{H}-\mathrm{PbI}_{2 .}(\mathrm{E}-\mathrm{H})$ show the Fourier transformations corresponding to the areas in the red squares in $(A-D)$, respectively, and confirm the dynamic radiation-induced transformation. Initially, the FT in (E) reveals a pristine cubic structure, but additional spots appear after brief exposure, circled in orange in (F). After further exposure, the additional spots disappear again, along with the $\{100\}$ spots, whose expected location is marked by red circles in $(F)$. Finally, after extended exposure, the FT contains two sets of spots: one set that can be indexed with the degraded cubic phase and another corresponding to the hexagonal $\mathrm{PbI}_{2}$-phase, marked with a blue and yellow circle in (G), respectively. The $\overline{2} 20_{c}\left(2.2493\right.$ A) and $110_{h}(2.2775$ A) spots overlap, indicating an intergrowth of the two phases. Indices marked with a subscript ' $c$ ' are from the epitaxial $\mathrm{FAPbI}_{3}$ phase and those marked with a subscript ' $h$ ' are from the hexagonal $\mathrm{PbI}_{2}$ phase. Note that the material is no longer in its pristine perovskite phase after the initial scan and the 'c' subscript only serves to illustrate the corresponding plane in the pristine phase. The scale bar represents $10 \mathrm{~nm}$ and applies to $(A-D)$.

\section{Nanoscopic properties of metal halide perovskites}

The implementation of low electron dose, $<200$ e $\AA^{-2}$, inevitably results in a low signal-tonoise ratio when images are recorded. We find that combining low-dose LAADF imaging with 
simple Butterworth and Bragg filters allows us to investigate pristine and slightly damaged $\mathrm{FAPbI}_{3}$ thin films with atomic resolution, observing phenomena invisible to other techniques. The details of each filtering technique are provided in the Methods section and in Fig. S5. Our findings are based on hundreds of STEM images taken across 7-8 thin-film samples prepared for each stoichiometry, with the selected images shown in the main section (and further examples provided in the Supplementary Material section) being carefully chosen to be representative of each material's microstructural features. In Fig. 3 (A-C) we show LAADF STEM images of polycrystalline perovskite films fabricated with excess $\mathrm{PbI}_{2}$. We observe a surprisingly coherent transition boundary between remnant precursor $\mathrm{PbI}_{2}$ (highlighted in light orange) and the $\mathrm{FAPbI}_{3}$ grains, with undetectable lattice misfit. In addition, a light and dark chequered pattern becomes clearly visible in images of $\mathrm{FAPbI}_{3}$ after mild radiation damage, as highlighted schematically in the bottom right of Fig. 3A through white and black circles and seen surrounding the $\mathrm{PbI}_{2}$ region in Fig. 3D. We propose that the observed chequered pattern in the central position of the $\mathrm{FAPbI}_{3}$ unit cell is triggered by an initially random, beam-induced loss of $\mathrm{FA}^{+}$, likely due to dissociation of the $\mathrm{FA}^{+}$by radiolysis $(42,43)$, potentially complemented by loss of I- through knock-on (displacement) effects $(41,44)$. We postulate that the remaining $\mathrm{FA}^{+}$ions then migrate to form an ordered structure with an associated energy reduction that helps stabilise the $\mathrm{FA}^{+}$deficient structure. Such migration of $\mathrm{FA}^{+}$ions within the perovskite structure after electron irradiation then results in the observed chequered pattern as an intermediate equilibrium(29). A degradation mechanism assuming solely the ordered loss of $\mathrm{I}^{-}$has previously been proposed based on analysis of diffraction patterns alone(30), however, we note that this approach struggles to distinguish between ordered loss of $\mathrm{FA}^{+}$and $\mathrm{I}^{-}$. Although vacancy ordering is clearly apparent on the $\mathrm{FA}^{+}$sublattice, there is no equivalent effect evident for the $\mathrm{I}$ or $\mathrm{Pb} / \mathrm{I}$ columns (see Fig. S8A for intensity line profiles corresponding to Fig. 3D). 
Our direct interpretation of real-space images is thus able to reveal that the degradation is strongly driven by the loss, and likely subsequent reordering, of $\mathrm{FA}^{+}$ions.

As further elaborated below, these microstructural features help explain important phenomena pertinent to perovskite solar cells. Specifically, small amounts of $\mathrm{PbI}_{2}$ are not detrimental to the solar cell performance(14-16) since perfect crystallographic alignment between the $\mathrm{PbI}_{2}$ and $\mathrm{FAPbI}_{3}$ perovskite occurs at the material interface, and no crystal defects are apparent in this region. The observation of the chequered pattern further explains why the perovskite structure can sustain significant deviations from stoichiometry and recover remarkably well from damage.
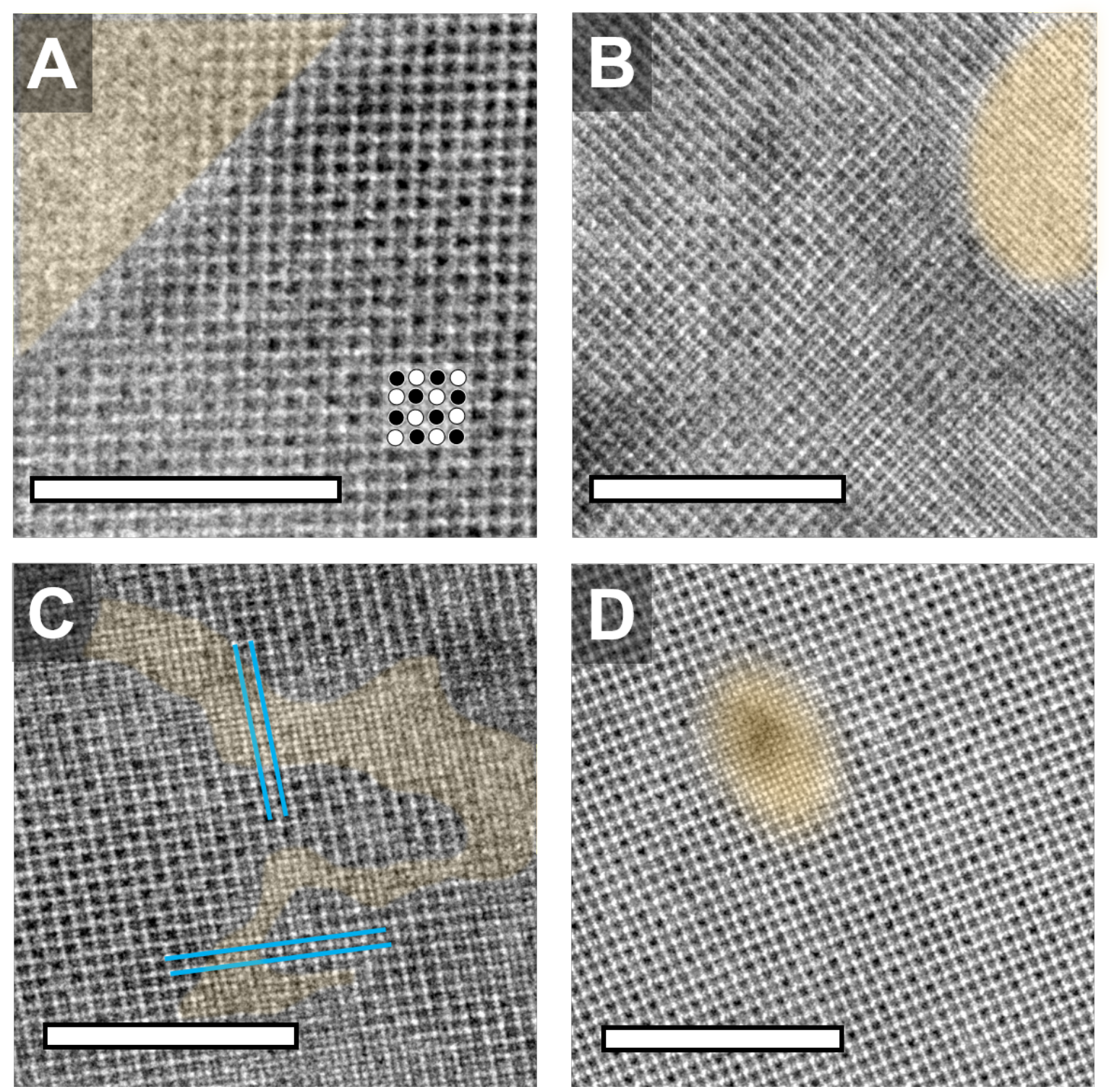
Fig. 3: Atomic-resolution $\mathrm{LAADF}$ micrographs of $\mathrm{FAPbI}_{3}$ thin films showing undistorted transitions between $\mathrm{PbI}_{2}$ (highlighted in light orange) and $\mathrm{FAPbI}_{3}$, as well as a chequered intensity after mild radiation damage. (A-C) show native intergrowth between $\mathrm{PbI}_{2}$ and $\mathrm{FAPbI}_{3}$ formed during the deposition process in 90-nm thick $\mathrm{FAPbI}_{3}$ films fabricated with excess $\mathrm{PbI}_{2}$, and (D) shows a local area of $\mathrm{PbI}_{2}$ formation, induced by focusing an electron beam on a near-stoichiometric $\mathrm{FAPbI}_{3}$ thin film. The highlighted area in (D) corresponds to the damage phase displayed in Fig. $2 C$ and the surrounding area corresponds to that shown in Fig. 2B. The transitions do not induce any measurable phase boundary misfit distortions in the crystal lattice. The nanoscopic $\mathrm{PbI}_{2}$ domain surrounded by $\mathrm{FAPbI}_{3}$ in (B) indicates that $\mathrm{PbI}_{2}$ can act as a seed for growth of $\mathrm{FAPbI}_{3}$ crystals. The parallel blue lines in $(\mathrm{C})$ begin in a $\mathrm{Pb} / \mathrm{I}$ lattice site and end in an $\mathrm{FA}^{+}$lattice site after crossing a $\mathrm{PbI}_{2}$ domain, indicating that areas on either side of the $\mathrm{PbI}_{2}$ are crystallographically separated and that the presence of $\mathrm{PbI}_{2}$ is not beam-induced. A chequered pattern in the intensity of the $\mathrm{FA}^{+}$lattice site is visible in (A,C,D), schematically presented as black and white circles in (A). This pattern is indicative of a highly ordered restructuring of the moieties in the crystal after mild electron radiation damage. The Fourier transform of (A) is seen in Fig. S6. All micrographs have been filtered using Butterworth filtering. The scale bars represent $10 \mathrm{~nm}$. The figure is shown without the overlaid colour graphics and with Fourier transforms in Fig. S7.

We note that at cryogenic temperatures, $\mathrm{MAPbI}_{3}$ has been shown to amorphise under electron irradiation rather than inducing the gradual, ordered restructuring of the crystal observed at room temperature(29). Rothmann et al. argued that such amorphisation at low temperatures is the result of impeded ion mobility(29), further supporting our notion here that ion migration is a key factor in mitigating the beam induced damage through atomic rearrangement. 
We further examine the nature of the native interfaces formed between $\mathrm{FAPbI}_{3}$ and its precursor $\mathrm{PbI}_{2}$ (Fig. 3). Small amounts of $\mathrm{PbI}_{2}$ surprisingly do not impede solar cell performance(14-16), but the reasons for such benign influence are still a matter of debate. We are able to reveal the presence of a low misfit, low lattice strain interface between $\mathrm{FAPbI}_{3}$ and $\mathrm{PbI}_{2}$ by examining the intergrowth between $\mathrm{FAPbI}_{3}$ and $\mathrm{PbI}_{2}$ in a film grown specifically under $\mathrm{PbI}_{2}$-rich conditions (see Methods). In Fig. 3B a nanoscopic domain of $\mathrm{PbI}_{2}$ with a continuous transition into $\mathrm{FAPbI}_{3}$ can be seen in the top right-hand corner, with the continuous transition likely arising because of the inclination of the interface to the beam direction. The $\mathrm{PbI}_{2}$ domain perfectly follows the surrounding perovskite structure and orientation. The lack of evidence for misfit dislocations (see Fig. S9 for more examples) shows that the interface is highly coherent and epitaxial. We note that the known $2 \mathrm{H}$ hexagonal phase of $\mathrm{PbI}_{2}$ could not be coherent with the cubic $\mathrm{FAPbI}_{3}$ in all directions in the interface, but that a modified $2 \mathrm{H}$ structure described in Fig. S10 can be coherent with the perovskite, and is possibly stabilised by its interface with $\mathrm{FAPbI}_{3}$ (see Fig. S10 for an atomic model) as evidenced by the square nature of the observed lattice. The weak van der Waals binding between the strongly ionically bound $\mathrm{PbI}_{2}$ sheets enables the existence of many different $\mathrm{PbI}_{2}$ polytypes(45), and a small shift between the layers of the $2 \mathrm{H}$ structure does allow coherent interfaces to form with $\mathrm{FAPbI}_{3}$ (see Fig. S10).(45) This coherence also indicates that the $\mathrm{PbI}_{2}$ has either acted as a seed for the surrounding perovskite or has grown out of the perovskite because of a local lack of FAI.

Further evidence for $\mathrm{PbI}_{2}$ seeding is revealed in Fig. 3C, where irregularly shaped domains of $\mathrm{PbI}_{2}$ can be seen to interweave with the perovskite lattice. Here, we observe a shift of half a unit cell in the perovskite crystal across the $\mathrm{PbI}_{2}$ domain, as evidenced by the two sets of parallel blue lines. These lines are oriented in the $<100>$ direction and begin in an $\mathrm{FA}^{+}$lattice site on one side of the $\mathrm{PbI}_{2}$ domain and end in a $\mathrm{Pb} / \mathrm{I}$ lattice site on the other side. These columns are not found together on the same $\{100\}$ plane in a perfect crystal so there must be a relative 
shift of half a unit cell on either side of the $\mathrm{PbI}_{2}$ region. The LAADF signal intensity line profile displayed in Fig. S8B further illustrates this shift across the $\mathrm{PbI}_{2}$ region. It is unlikely for degradation of $\mathrm{FAPbI}_{3}$ to have resulted in the growth of such an epitaxial $\mathrm{PbI}_{2}$ phase which exhibits a half-unit-cell distortion of the $\mathrm{FAPbI}_{3}$ matrix, because the low misfit of the interface would not give a driving force for such a distortion of the $\mathrm{FAPbI}_{3}$ structure. We can therefore conclude that the perovskite has grown out of the $\mathrm{PbI}_{2}$ and not vice versa. Despite this shift, geometric phase analysis (GPA) strain mapping, displayed in Fig. S11, shows an absence of significant strain associated with this perovskite/ $\mathrm{PbI}_{2}$ interface. Fig. $\mathrm{S} 12 \mathrm{~A}, \mathrm{~B}$ show examples of both the half a unit cell shift and the $\mathrm{PbI}_{2}$ intergrowth, respectively, similarly occurring in pristine perovskite that has not suffered electron-beam damage. These observations provide further evidence that the presence of perovskite/ $\mathrm{PbI} 2$ intergrowth is not caused by radiationinduced damage, but is an intrinsic property of the material, present from the point of crystal growth. In order to verify that this approach is also applicable to other hybrid perovskites, we also imaged $\mathrm{PbI}_{2}$-rich $\mathrm{MAPbI}_{3}$ and found similar crystallographic properties, as shown in Fig. S13.

\section{Grain boundaries and crystal defects}

We further reveal the nature of the boundaries between individual $\mathrm{FAPbI}_{3}$ grains through highresolution LAADF imaging. Such grain boundaries are of particular interest for perovskite solar cells since they have been proposed to act both as charge recombination centres $(18,46$, 47) and conduction pathways(16). In addition, grain boundaries have been shown to be intimately linked with the long-term stability of MHPs such as $\mathrm{FAPbI}_{3}(22)$ and the photostability of mixed-halide perovskites towards halide segregation(23). Therefore, much effort has been devoted to passivating their negative impact and promoting their positive influence, through appropriate treatments and film growth $(11,16,19,48-51)$. While many techniques have been used to infer properties of the grain boundaries in photoactive perovskites $(11,17)$, 
reliable imaging with atomic resolution has remained elusive, which has led to some uncertainty about the inherent nature of the boundaries $(17,51)$. A full understanding of the crystallographic nature of grain boundaries is likely to aid an understanding of a plethora of observations already well established in the field of perovskite solar cell devices, leading to further improvements in performance.

Butterworth-filtered LAADF images displayed in Fig. 4A-C illustrate a representative set of grain boundaries in an evaporated thin film of $\mathrm{FAPbI}_{3}$, with several striking features. First, the grains are highly crystalline adjacent to the boundaries, showing that the presence of the boundaries does not disrupt the long-range crystal quality of the surrounding perovskite. Secondly, the grain boundaries themselves are clearly defined, with the perovskite structure remaining unchanged to the interface, with no obvious preferred interfacial orientation and typically no amorphous material visible. These two points are illustrated by the strain maps displayed in Fig. S16. While an exhaustive description of the strain associated with all the potential boundaries is beyond the scope of this study, we have mapped the strain associated with the low-angle grain boundary seen in the bottom left of Fig. 4B. We find that the strain is negligible to within one or two unit cells away from the boundary. Along the boundary itself, the strain exhibits a periodic behavior with alternating sign, which arises from the periodic array of edge dislocations forming the grain boundary. The yy strain (lying close to perpendicular to the dislocation burgers vectors) is much lower at the boundary.

Near- $120^{\circ}$ triple boundaries are commonly observed at the intersection of three grains. Fig. $4 \mathrm{~A}$ shows a typical image of a triple boundary in a $\mathrm{FAPbI}_{3}$ film, which we generally found to be crystallographically continuous, with minimal lattice distortion and no defects extending away from the grain boundary plane associated with them. Across the STEM micrographs examined, we found this type of "clean" triple boundary to be the most common in $\mathrm{FAPbI}_{3}$, with Fig. S14 
МIAAAS

providing further examples, as well as an illustration of the wide range of grain boundary angles found within the films. 

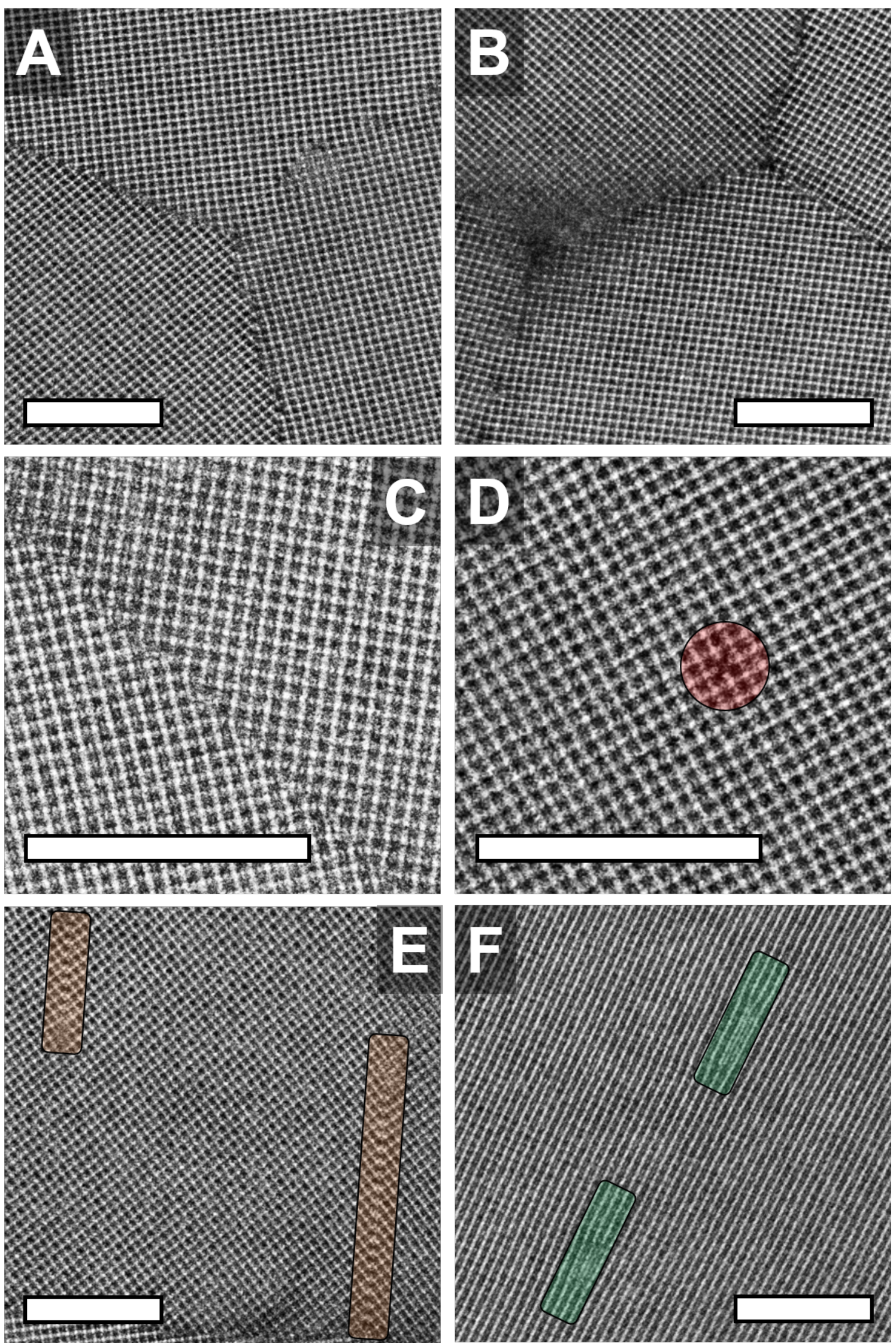
Fig. 4: Butterworth-filtered atomic-resolution $L A A D F$ micrographs of representative $\mathrm{FAPbI}_{3}$ grain boundaries and crystal defects as seen in a 30-nm thick film prepared close to the correct stoichiometry. (A) Abrupt grain boundaries meeting at a triple junction showing no amorphous or intergranular phase. (B) A triple junction showing signs of amorphisation in the left-hand intersection and a line of vacancies at the right-hand intersection. The boundaries appear randomly oriented, with the presence of a curved boundary in (B) visible in the top right-hand corner. The low-angle grain boundary in the lower left of the image are formed of a series of $<100>$ edge dislocations. (C) A straight section of a grain boundary with virtually no empty lattice sites and very little distortion across the boundary. (D) Aligned vacancy defects on the I/Pb lattice site are seen in the red circle. (E) Intrinsic stacking faults are seen in the orange rectangles with a stacking fault vector of $1 / 2<110>$. (F) Edge dislocations are seen in the green rectangles with a burgers vector of $\langle 100\rangle$. The dislocations can be seen to have dissociated in the climb direction forming two $1 / 2<100>$ partial dislocations and a fault lying perpendicular to the glide plane. The scale bars represent $10 \mathrm{~nm}$. The figure is shown without the coloured highlights in Fig. S15.

Interestingly, while most grain boundaries in these evaporated $\mathrm{FAPbI}_{3}$ films appear to be highly ordered and sharp, some exceptions also occur. For example, the triple boundary displayed in Fig. 4B contains amorphous material and aligned point defects, as seen in the left-hand and right-hand sides, respectively. Such variation in the nature of grain boundaries observed here for $\mathrm{FAPbI}_{3}$ may thus also explain the disparity in the grain boundary properties reported in $\mathrm{MAPbI}_{3}$, ranging from detrimental $(18,46,47)$ to beneficial $(16)$, or even $\operatorname{both}(11,17)$. If a particular preparation method favors a particularly beneficial or detrimental type of grain boundary it can improve or decrease solar cell performance, respectively. 
Finally, we uncover the presence of aligned point defects and stacking faults in $\mathrm{FAPbI}_{3}$. The nature and distribution of crystal defects in photoactive perovskites is a topic which has received significant attention, but the exact nature of these defects is still unclear(41). Most studies and simulations have focused on point defects and their influence on charge-carrier properties(52). As revealed in Fig. 4D, aligned point defects in the form of vacancies on the $\mathrm{FA}^{+}$sub-lattice are indeed present in the $\mathrm{FAPbI}_{3}$ crystal. We find here that stacking faults and edge dislocations, displayed in Fig. 4E,F, respectively, are also very common in $\mathrm{FAPbI}_{3}$. Interestingly, the stacking faults shown in Fig. 4E correspond to a shift of half a unit cell, connecting $\mathrm{Pb}-\mathrm{I}$ columns with $\mathrm{I}^{-}$columns rather than with $\mathrm{FA}^{+}$columns. Edge dislocations are also present, as highlighted in the green rectangles in Fig. 4F. The dislocations are dissociated in a direction perpendicular to their glide plane, and are thus referred to as climb dissociated, with an apparent region of $\mathrm{PbI}_{2}$ forming the planar fault joining the dislocations.

\section{Discussion}

Resolving the atomic structure of $\mathrm{FAPbI}_{3}$ thin films allows us to explain many of the intriguing and unique properties of MHPs that had already been well established in the literature, but lacked an explanation to date. The observed loss of $\mathrm{FA}^{+}$through an intermediate perovskite structure displaying a chequered $\mathrm{FA}^{+}$vacancy pattern provides a direct clue to the ability for metal halide perovskites to sustain significant deviations in stoichiometry, and the regenerative capacity of hybrid organic-inorganic MHPs. This mechanism is likely to be general, given that gradual changes have also been observed in photoluminescence and cathodoluminescence spectra when MHPs are exposed to photon(53) and electron(54) irradiation, respectively, which do not occur in all-inorganic photoactive perovskites(54). It is also bound to underpin the readily regenerative properties of photobleached $\mathrm{MAPbI}_{3}$ solar cells when they are exposed to gaseous $\operatorname{MAI}(13)$, showing that the lead-iodide perovskite framework remains even after a significant loss of the organic A-cation. This highly adaptable and resilient structure can thus 
accommodate dramatic stoichiometry changes without collapsing, which, along with its potential for regeneration, is a clear advantage for applications. Such resilience of structure, induced by ion migration, may well be unique to metal halide perovskites, as compared to other more covalent crystalline solids, and is likely to be the origin of the "radiation hardness" of metal halide perovskites, beckoning well for their use in space applications and as gamma and X-ray detectors.

In addition, the observed atomically coherent nature of perovskite/ $\mathrm{PbI}_{2}$ interfaces explains how the presence of $\mathrm{PbI}_{2}$ can enhance the crystal quality of MHP films(16) by acting as seeds for crystal growth, while not adversely affecting the local optoelectronic properties of the material. Higher-energy charge carriers generated in $\mathrm{PbI}_{2}$ can easily transfer to the lower-energy perovskite, contributing to the overall photogenerated current(55) which is relatively unaffected by the perovskite/ $\mathrm{PbI}_{2}$ boundaries. The highly ordered nature of the perovskite/ $\mathrm{PbI}{ }_{2}$ boundary is therefore a core mechanism underpinning the facile fabrication of highperformance solar cells. In essence, this feature forms the MHP analogue of the native $\mathrm{SiO}_{2}-\mathrm{Si}$ interface, which is benign and is probably the main reason why our electronics industry is primarily based on silicon.

The presence of predominantly sharp, strain-free boundaries between individual $\mathrm{FAPbI}_{3}$ grains is a vital clue to the reasons for the high performance of perovskite solar cells based on polycrystalline films. Grain boundaries in other polycrystalline semiconductor solar cells are generally detrimental to performance, decreasing the short-circuit current density and introducing trap states $(56,57)$. The revelation of such sharp boundaries in evaporated $\mathrm{FAPbI}_{3}$ films, with some amorphous exceptions, therefore presents a first step towards understanding the exact nature of grain boundaries in perovskite solar cells, through which improved control can ensure the highest possible solar cell performance and stability. In addition, our acquisition of truly atomic-resolution images, enabled through careful choices of image acquisition and 
filtering approaches, will form the basis of investigations into the microstructure of MHPs across a wide range of different stoichiometries. For example, addition of small fractions of Cs to replace some of the FA cations in $\mathrm{FAPbI}_{3}$ has been shown to enhance material stability and crystallinity (58), which may link with the nature of grain boundaries.

Finally, stacking faults have been shown to act as recombination sites in dislocation-free silicon(59), and edge dislocations decrease the PL intensity in multicrystalline silicon(60). Our identification of aligned point defects in the form of vacancies on the $\mathrm{FA}^{+}$sub-lattice and climbdissociated dislocations in $\mathrm{FAPbI}_{3}$ provides first insights into the specific forms such defects take in MHPs. Such information will pave the way for future modelling of defects based on first principles, which can reveal the impact such defects have on the optoelectronic properties of metal halide perovskites, and the subsequent performance of perovskite solar cells.

In conclusion, using low-dose LAADF STEM imaging, we are able to obtain atomic-resolution micrographs of pristine cubic $\mathrm{FAPbI}_{3}$ films. We show that electron irradiation causes the perovskite structure to change through an initial loss of the $\mathrm{FA}^{+}$ion, leaving behind a resilient, partially empty but ordered perovskite lattice, which deteriorates to $\mathrm{PbI}_{2}$ under further irradiation. This intermediate step explains the surprising regenerative properties of radiationdamaged perovskite films, highlighting the highly adaptive nature of the perovskite structure. We further examine the film microstructure, showing that $\mathrm{PbI}_{2}$ precursor remnants readily and seamlessly intergrow with the perovskite lattice, with an atomically continuous co-existence of the two materials within grains. We image $\mathrm{FAPbI}_{3}$ grain boundaries, revealing that the longrange perovskite structure is preserved up to the grain boundaries, where sharp interfaces are generally present, and finally we identify a number of different crystal defects in the perovskite lattice. These findings reveal several mechanisms underpinning the remarkable performance of metal halide perovskites and pave the way for obtaining atomically localised information 
from metal halide perovskites, enabling control over these materials for optoelectronic and solar energy applications at a hitherto unseen level.

\section{Methods and Materials}

\section{Sample preparation}

Both $\mathrm{FAPbI}_{3}$ and $\mathrm{MAPbI}_{3}$ thin films were deposited on the carbon-coated side of 300 -mesh copper TEM grids. Immediately prior to deposition, the grids were $\mathrm{O}_{2}$-plasma cleaned for 0.3 minutes.

$\mathrm{CH}(\mathrm{NH})_{2} \mathrm{I}(\mathrm{FAI})$ and $\mathrm{PbI}_{2}$ were co-evaporated in a customised thermal evaporation chamber described previously $(61)$. Heating of the sources started once the chamber pressure fell below $5 \times 10^{-6}$ mbar. The $\mathrm{PbI}_{2}$ rate was kept constant at $0.23 \AA / \mathrm{s}$, measured using a gold-plated quartz microbalance (QMB). For the " $\mathrm{PbI}_{2}$-rich" $\mathrm{FAPbI}_{3}$ films with significant excess of $\mathrm{PbI}_{2}$, the FAI rate was set to $40 \%$ of the rate used for the "near-stoichiometric" $\mathrm{FAPbI}_{3}$ films. This resulted in FAI crucible temperatures of around $150{ }^{\circ} \mathrm{C}$ during the deposition of the $\mathrm{PbI}_{2}$-rich $\mathrm{FAPbI}_{3}$

films and $180{ }^{\circ} \mathrm{C}$ during the deposition of the near-stoichiometric $\mathrm{FAPbI}_{3}$ films. During deposition, the pressure increased to $1-2 \times 10^{-5}$ mbar for the $\mathrm{PbI}_{2}$-rich $\mathrm{FAPbI}_{3}$ films, and to 5$9 \times 10^{-5}$ mbar for the near-stoichiometric $\mathrm{FAPbI}_{3}$ films. The duration of the overall perovskite deposition was controlled such that the film thickness was $90 \mathrm{~nm}$ for the $\mathrm{PbI}_{2}$-rich $\mathrm{FAPbI}_{3}$ films and $30 \mathrm{~nm}$ for the near-stoichiometric $\mathrm{FAPbI}_{3}$ films. After deposition, all films were annealed at $170{ }^{\circ} \mathrm{C}$ for $1 \mathrm{~min}$. The films were stored in a nitrogen-filled glove box after preparation and transported to the microscope in a triple-sealed nitrogen atmosphere to prevent exposure to moisture. 
$\mathrm{CH}_{3} \mathrm{NH}_{3} \mathrm{PbI}_{3}\left(\mathrm{MAPbI}_{3}\right)$ thin films were prepared using a recently published method(32). $\mathrm{CH}_{3} \mathrm{NH}_{3} \mathrm{I}$ (MAI, Greatcell) and $\mathrm{PbI}_{2}$ (99.999\% metal base, Sigma-Aldrich) were co-evaporated in the same custom Lesker thermal evaporator chamber. Heating of the sources started once the chamber pressure fell below $5 \times 10^{-6}$ mbar. During the subsequent evaporation, the pressure increased to $1-2 \times 10^{-5}$ mbar. The $\mathrm{PbI}_{2}$ rate was kept constant at $0.30 \AA / \mathrm{s}$ using a gold-plated quartz microbalance, while the MAI temperature was controlled to aim for an overall $\mathrm{MAPbI}_{3}$ deposition rate of $0.45 \AA / \mathrm{s}$, as measured by a quartz microbalance located next to the substrate. For the depositions used in this work, this involved ramping the MAI crucible from $175.1{ }^{\circ} \mathrm{C}$ to $175.6{ }^{\circ} \mathrm{C}$ over $1070 \mathrm{~s}$, for $1.1 \mathrm{~g}$ of MAI initially in the crucible. The substrate was cooled to $0{ }^{\circ} \mathrm{C}$ during the deposition; the sensors were cooled to $17^{\circ} \mathrm{C}$.

All samples were loaded rapidly into the microscope, being in contact with air for less than a minute.

Perovskite film thicknesses were determined from calibration of material deposition rates of the two sources according to quartz microbalance readings inside the vacuum chamber, supported by film depositions on hard transparent quartz substrates for which film thickness readings were determined from a combination of Dektak profilometer readings and optical transmission measurements.

\section{Image acquisition}

Microscopy for imaging and condition development was performed on two microscopes. A JEOL ARM-200F cold FEG, $\mathrm{C}_{\mathrm{s}}$ probe corrected STEM at 80 or $200 \mathrm{kV}$ acceleration voltage, 20-21 mrad convergence angle, using an annular dark field detector at $8 \mathrm{~cm}$ camera length, 
resulting in inner and outer collection angles of 33 and $120.77 \mathrm{mrad}$, respectively for LAADF imaging and 72.80 and $235.75 \mathrm{mrad}$ for HAADF imaging. A JEOL ARM-300F $\mathrm{C}_{\mathrm{s}}$ probe corrected STEM at $300 \mathrm{kV}$ was used for imaging condition development. Both room temperature and cryogenic conditions were examined, and the cryogenic conditions did not noticeably reduce the beam sensitivity of the $\mathrm{FAPbI}_{3}$ thin films, so all imaging was done at room temperature. Similarly, no noticeable difference in beam sensitivity was found between 200 and $300 \mathrm{kV}$ acceleration voltage, but the material was found to damage faster at $80 \mathrm{kV}$. All imaging presented in the manuscript was at $200 \mathrm{kV}$. All alignments and focusing were done away from the areas imaged to reduce electron beam-induced damage to the material. All micrographs were obtained without tilting the sample to reduce the beam damage.

The specific image acquisition conditions for all images are listed in Table S1.

\section{References}

1.H.-S. Kim et al., Lead iodide perovskite sensitized all-solid-state submicron thin film mesoscopic solar cell with efficiency exceeding 9\%. Scientific Reports 2, 591 (2012).

2.M. Liu, M. B. Johnston, H. J. Snaith, Efficient planar heterojunction perovskite solar cells by vapour deposition. Nature 501, 395 (2013).

3.H. Zhou et al., Interface engineering of highly efficient perovskite solar cells. Science $\mathbf{3 4 5}$, 542-546 (2014).

4.N. J. Jeon et al., Solvent engineering for high-performance inorganic-organic hybrid perovskite solar cells. Nat Mater 13, 897-903 (2014).

5.J.-P. Correa-Baena et al., The rapid evolution of highly efficient perovskite solar cells. Energy \& Environmental Science 10, 710-727 (2017).

6.M. Saliba et al., Incorporation of rubidium cations into perovskite solar cells improves photovoltaic performance. Science 354, 206-209 (2016).

7.D. P. McMeekin et al., A mixed-cation lead mixed-halide perovskite absorber for tandem solar cells. Science 351, 151-155 (2016).

8.D. W. DeQuilettes et al., Photo-induced halide redistribution in organic-inorganic perovskite films. Nature communications 7, 1-9 (2016).

9.C. C. Stoumpos, C. D. Malliakas, M. G. Kanatzidis, Semiconducting tin and lead iodide perovskites with organic cations: phase transitions, high mobilities, and near-infrared photoluminescent properties. Inorganic chemistry 52, 9019-9038 (2013). 
10.T. Baikie et al., A combined single crystal neutron/X-ray diffraction and solid-state nuclear magnetic resonance study of the hybrid perovskites $\mathrm{CH}_{3} \mathrm{NH}_{3} \mathrm{PbX}_{3}(\mathrm{X}=\mathrm{I}, \mathrm{Br}$ and $\mathrm{Cl})$. Journal of Materials Chemistry A 3, 9298-9307 (2015).

11.M. U. Rothmann, W. Li, J. Etheridge, Y. B. Cheng, Microstructural Characterisations of Perovskite Solar Cells-From Grains to Interfaces: Techniques, Features, and Challenges. Advanced Energy Materials 7, (2017).

12.K. Domanski, E. A. Alharbi, A. Hagfeldt, M. Grätzel, W. Tress, Systematic investigation of the impact of operation conditions on the degradation behaviour of perovskite solar cells. Nature Energy 3, 61-67 (2018).

13.L. Hong et al., Improvement and Regeneration of Perovskite Solar Cells via Methylamine Gas Post-Treatment. Advanced Functional Materials 27, 1703060 (2017).

14.D. H. Cao et al., Remnant $\mathrm{PbI}_{2}$, an unforeseen necessity in high-efficiency hybrid perovskite-based solar cells? Apl Materials 2, 091101 (2014).

15.H.-Y. Hsu et al., Optimization of $\mathrm{PbI}_{2} / \mathrm{MAPbI}_{3}$ perovskite composites by scanning electrochemical microscopy. The Journal of Physical Chemistry C 120, 19890-19895 (2016).

16.Y. C. Kim et al., Beneficial effects of $\mathrm{PbI}_{2}$ incorporated in organo-lead halide perovskite solar cells. Advanced Energy Materials 6, 1502104 (2016).

17.J.-W. Lee et al., The role of grain boundaries in perovskite solar cells. Materials Today Energy 7, 149-160 (2018).

18.R. Long, J. Liu, O. V. Prezhdo, Unravelling the effects of grain boundary and chemical doping on electron-hole recombination in $\mathrm{CH}_{3} \mathrm{NH}_{3} \mathrm{PbI}_{3}$ perovskite by time-domain atomistic simulation. Journal of the American Chemical Society 138, 3884-3890 (2016).

19.T. Niu et al., Stable High-Performance Perovskite Solar Cells via Grain Boundary Passivation. Advanced Materials 30, 1706576 (2018).

20.S. Jariwala et al., Local crystal misorientation influences non-radiative recombination in halide perovskites. Joule 3, 3048-3060 (2019).

21.C. L. Davies et al., Impact of the organic cation on the optoelectronic properties of formamidinium lead triiodide. The journal of physical chemistry letters 9, 4502-4511 (2018).

22.S. K. Yadavalli et al., Mechanisms of Exceptional Grain Growth and Stability in Formamidinium Lead Triiodide Thin Films for Perovskite Solar Cells. Acta Materialia, (2020).

23.A. Knight, L. M. Herz, Preventing phase segregation in mixed-halide perovskites: a perspective. Energy \& Environmental Science, (2020).

24.O. Breitenstein, J. Rakotoniaina, M. H. Al Rifai, M. Werner, Shunt types in crystalline silicon solar cells. Progress in Photovoltaics: Research and Applications 12, 529-538 (2004).

25.A. Stoffers et al., Complex Nanotwin Substructure of an Asymmetric $\Sigma 9$ Tilt Grain Boundary in a Silicon Polycrystal. Physical review letters 115, 235502 (2015).

26.Y. Yu et al., Atomic resolution imaging of halide perovskites. Nano letters 16, 7530-7535 (2016).

27.D. Zhang et al., Atomic-resolution transmission electron microscopy of electron beamsensitive crystalline materials. Science 359, 675-679 (2018).

28.Y. Li et al., Unravelling degradation mechanisms and atomic structure of organic-inorganic halide perovskites by cryo-EM. Joule 3, 2854-2866 (2019).

29.M. U. Rothmann et al., Structural and chemical changes to $\mathrm{CH}_{3} \mathrm{NH}_{3} \mathrm{PbI}_{3}$ induced by electron and gallium ion beams. Advanced Materials 30, 1800629 (2018). 
30.S. Chen et al., Atomic scale insights into structure instability and decomposition pathway of methylammonium lead iodide perovskite. Nature communications 9, 1-8 (2018).

31.R. Hooley, A. Brown, A. Kulak, F. Meldrum, R. Brydson, in Journal of Physics: Conference Series. (IOP Publishing, 2017), vol. 902, pp. 012005.

32.K. B. Lohmann et al., Control over crystal size in vapor deposited metal-halide perovskite films. ACS Energy Letters 5, 710-717 (2020).

33.J. Borchert et al., Large-area, highly uniform evaporated formamidinium lead triiodide thin films for solar cells. ACS Energy Letters 2, 2799-2804 (2017).

34.J. Gonnissen et al., Optimal experimental design for the detection of light atoms from highresolution scanning transmission electron microscopy images. Applied Physics Letters 105, 063116 (2014).

35.L. Oesinghaus et al., Toward tailored film morphologies: the origin of crystal orientation in hybrid perovskite thin films. Advanced Materials Interfaces 3, 1600403 (2016).

36.H. J. Jung et al., Stability of halide perovskite solar cell devices: in situ observation of oxygen diffusion under biasing. Advanced Materials 30, 1802769 (2018).

37.T. W. Kim et al., Self-Organized Superlattice and Phase Coexistence inside Thin Film Organometal Halide Perovskite. Advanced Materials 30, 1705230 (2018).

38.Z. Chen, Z.-G. Gu, W.-Q. Fu, F. Wang, J. Zhang, A confined fabrication of perovskite quantum dots in oriented MOF thin film. ACS applied materials \& interfaces $\mathbf{8}, 28737$ 28742 (2016).

39.M. Xiao et al., A Fast Deposition-Crystallization Procedure for Highly Efficient Lead Iodide Perovskite Thin-Film Solar Cells. Angewandte Chemie 126, 10056-10061 (2014).

40.Y. Zhou, H. Sternlicht, N. P. Padture, Transmission electron microscopy of halide perovskite materials and devices. Joule 3, 641-661 (2019).

41.J. Ran et al., Electron-Beam-Related Studies of Halide Perovskites: Challenges and Opportunities. Advanced Energy Materials, 1903191 (2020).

42.R. Egerton, Radiation damage to organic and inorganic specimens in the TEM. Micron 119, 72-87 (2019).

43.R. F. Egerton, P. Li, M. Malac, Radiation damage in the TEM and SEM. Micron 35, 399409 (2004).

44.J. Ran et al., Electron-Beam-Related Studies of Halide Perovskites: Challenges and Opportunities. Advanced Energy Materials, 10, 1903191 (2020).

45.P. A. Beckmann, A review of polytypism in lead iodide. Crystal Research and Technology 45, 455-460 (2010).

46.H.-S. Duan et al., The identification and characterization of defect states in hybrid organicinorganic perovskite photovoltaics. Physical chemistry chemical physics 17, 112-116 (2015).

47.X. Wen et al., Defect trapping states and charge carrier recombination in organic-inorganic halide perovskites. Journal of Materials Chemistry C 4, 793-800 (2016).

48.D. W. de Quilettes et al., Impact of microstructure on local carrier lifetime in perovskite solar cells. Science 348, 683-686 (2015).

49.Q. Chen et al., Controllable self-induced passivation of hybrid lead iodide perovskites toward high performance solar cells. Nano letters 14, 4158-4163 (2014).

50.Y. Shao, Z. Xiao, C. Bi, Y. Yuan, J. Huang, Origin and elimination of photocurrent hysteresis by fullerene passivation in $\mathrm{CH}_{3} \mathrm{NH}_{3} \mathrm{PbI}_{3}$ planar heterojunction solar cells. Nature communications 5, 1-7 (2014).

51.A. F. Castro-Méndez, J. Hidalgo, J. P. Correa-Baena, The role of grain boundaries in perovskite solar cells. Advanced Energy Materials 9, 1901489 (2019). 
52.J. M. Ball, A. Petrozza, Defects in perovskite-halides and their effects in solar cells. Nature Energy 1, 1-13 (2016).

53.A. Merdasa et al., Super-resolution luminescence microspectroscopy reveals the mechanism of photoinduced degradation in $\mathrm{CH}_{3} \mathrm{NH}_{3} \mathrm{PbI}_{3}$ perovskite nanocrystals. The Journal of Physical Chemistry C 120, 10711-10719 (2016).

54.C. Xiao et al., Mechanisms of Electron-Beam-Induced Damage in Perovskite Thin Films Revealed by Cathodoluminescence Spectroscopy. The Journal of Physical Chemistry C 119, 26904-26911 (2015).

55.J. B. Patel et al., Photocurrent spectroscopy of perovskite solar cells over a wide temperature range from 15 to $350 \mathrm{~K}$. The journal of physical chemistry letters 9, 263-268 (2018).

56.J. D. Zook, Effects of grain boundaries in polycrystalline solar cells. Applied Physics Letters 37, 223-226 (1980).

57.F. Greuter, G. Blatter, Electrical properties of grain boundaries in polycrystalline compound semiconductors. Semiconductor Science and Technology 5, 111 (1990).

58.J.-W. Lee et al., Formamidinium and Cesium Hybridization for Photo- and Moisture-Stable Perovskite Solar Cell. Advanced Energy Materials 5, 1501310 (2015).

59.L. Kimerling, H. Leamy, J. Patel, The electrical properties of stacking faults and precipitates in heat-treated dislocation-free Czochralski silicon. Applied Physics Letters 30, 217219 (1977).

60.T. W. Kim et al., Self-Organized Superlattice and Phase Coexistence inside Thin Film Organometal Halide Perovskite. Advanced Materials 30, 1705230 (2018).

61.J. Borchert et al., Impurity Tracking Enables Enhanced Control and Reproducibility of Hybrid Perovskite Vapor Deposition. ACS applied materials \& interfaces 11, 2885128857 (2019).

We thank the David Cockayne Centre for Electron Microscopy, University of Oxford, for access and support in the use of the JEOL ARM200F instrument (proposal number EP/K040375/1). We also thank Diamond Light Source for access and support in use of the electron Physical Science Imaging Centre (E02, MG21734) that contributed to the results presented here. L.M.H and M.B.J. thank the Humboldt Foundation for Research Awards.

\section{Funding:}

The authors gratefully acknowledge financial support from the Engineering and Physical Sciences Research Council (UK) (EPSRC) through grant number EP/P033229/1 and through the EPSRC CDT for New and Sustainable Photovoltaics. 


\section{Author contributions}

M.U.R. designed and carried out the electron microscope studies, and analysed and interpreted data. J.S.K. participated in the EM studies and provided insights in data interpretation and during editing. J.B and K.L. prepared the samples. C.O.L. and A.S. assisted with the EM studies. L.C. performed the multislice simulations. H.J.S. contributed to data analysis and interpretation. M.B.J., P.D.N., and L.M.H. designed the study, interpreted data, and supervised M.U.R., who wrote the manuscript with input from all co-authors.

\section{Competing interests declaration}

The authors declare the following competing financial interest: H.J.S. is the Chief Scientific Officer and a Director of Oxford PV Ltd, a company that is commercializing perovskite photovoltaic technologies.

\section{Correspondence}

Correspondence and requests for materials should be addressed to L.M.H. or P.D.N.

\section{Data availability}

The datasets and numerical analysis sets described in this manuscript are available for download from the Oxford University Research Archive, under the following DOI: [DOI will be supplied upon acceptance to ensure file names correctly match final figure names].

\section{Supplementary Materials:}

Figures S1-S16

Table S1

References 


\section{Science \\ $\triangle$ IAAAS}

\section{Supplementary Materials for}

\section{Atomic-scale microstructure of metal halide perovskite}

Authors: Mathias Uller Rothmann ${ }^{1}$, Judy S. Kim ${ }^{2,3,4}$, Juliane Borchert ${ }^{1}$, Kilian Lohmann ${ }^{1}$, Colum M. O'Leary ${ }^{2}$, Alex Sheader ${ }^{2}$, L. Clark ${ }^{2}$, Henry J. Snaith ${ }^{1}$, Michael B. Johnston ${ }^{1}$, Peter D. Nellist ${ }^{2}$, and Laura M. Herz ${ }^{1 *}$

\section{Affiliations:}

${ }^{1}$ Department of Physics, University of Oxford, Clarendon Laboratory, Parks Road, Oxford OX1 3PU, UK

${ }^{2}$ Department of Materials, University of Oxford, Parks Road, Oxford OX1 3PH, UK ${ }^{3}$ ePSIC, Diamond Light Source, Harwell, Didcot, OX11 0DE, UK

${ }^{4}$ Rosalind Franklin Institute, Fermi Ave, Harwell, Didcot, OX11 0QS, UK

*Correspondence to: laura.herz@physics.ox.ac.uk

This PDF file includes:

Materials and Methods

Figs. S1 to S16

Table S1 
Table S1: Overview of the imaging conditions for the images included in this manuscript.

\begin{tabular}{|c|c|c|c|c|}
\hline Figure & Beam current & Dwell-time & Pixel size & $\begin{array}{l}\text { Total dose } \\
\text { per area } \\
\text { per scan }\end{array}$ \\
\hline $1 \mathrm{~A}-\mathrm{B}$ & $1.49 \mathrm{pA}$ & $1 \mu \mathrm{s}$ & $0.42 \AA \times 0.42 \AA$ & 105 e $\AA^{-2}$ \\
\hline $2 \mathrm{~A}-\mathrm{C}$ & $1.49 \mathrm{pA}$ & $2 \mu \mathrm{s}$ & $0.53 \AA \times 0.53 \AA$ & 66 e $\AA^{-2}$ \\
\hline $3 \mathrm{~A}-\mathrm{C}$ & $1.49 \mathrm{pA}$ & $2 \mu \mathrm{s}$ & $0.35 \AA \times 0.35 \AA$ & 152 e $\AA^{-2}$ \\
\hline $3 \mathrm{D}$ & $1.49 \mathrm{pA}$ & $2 \mu \mathrm{s}$ & $0.29 \AA \times 0.29 \AA$ & 221 e $\AA^{-2}$ \\
\hline 4A,B,E,F & $1.49 \mathrm{pA}$ & $2 \mu \mathrm{s}$ & $0.29 \AA \times 0.29 \AA$ & 221 e $\AA^{-2}$ \\
\hline $4 \mathrm{C}$ & $1.49 \mathrm{pA}$ & $2 \mu \mathrm{s}$ & $0.42 \AA \times 0.42 \AA$ & 105 e $\AA^{-2}$ \\
\hline $4 \mathrm{D}$ & $1.49 \mathrm{pA}$ & $2 \mu \mathrm{s}$ & $0.53 \AA \times 0.53 \AA$ & 66 e $\AA^{-2}$ \\
\hline S2A-C & $1.49 \mathrm{pA}$ & $1 \mu \mathrm{s}$ & $0.42 \AA \times 0.42 \AA$ & 105 e $\AA^{-2}$ \\
\hline S3A-C & $1.49 \mathrm{pA}$ & $2 \mu \mathrm{s}$ & $0.53 \AA \times 0.53 \AA$ & 66 e $\AA^{-2}$ \\
\hline S4A & $1.49 \mathrm{pA}$ & $1 \mu \mathrm{s}$ & $0.42 \AA \times 0.42 \AA$ & 105 e $\AA^{-2}$ \\
\hline S5A-F & $1.49 \mathrm{pA}$ & $2 \mu \mathrm{s}$ & $0.35 \AA \times 0.35 \AA$ & 152 e $\AA^{-2}$ \\
\hline S7A-C & $1.49 \mathrm{pA}$ & $2 \mu \mathrm{s}$ & $0.35 \AA \times 0.35 \AA$ & 152 e $\AA^{-2}$ \\
\hline S7D & $1.49 \mathrm{pA}$ & $2 \mu \mathrm{s}$ & $0.29 \AA \times 0.29 \AA$ & 221 e $\AA^{-2}$ \\
\hline S9A-C & $1.49 \mathrm{pA}$ & $2 \mu \mathrm{s}$ & $0.53 \AA \times 0.53 \AA$ & 66 e $\AA^{-2}$ \\
\hline S12A-B & $1.49 \mathrm{pA}$ & $2 \mu \mathrm{s}$ & $0.53 \AA \times 0.53 \AA$ & 66 e $\AA^{-2}$ \\
\hline S13 & $1.49 \mathrm{pA}$ & $2 \mu \mathrm{s}$ & $0.53 \AA \times 0.53 \AA$ & 66 e $\AA^{-2}$ \\
\hline S14A-C & $1.49 \mathrm{pA}$ & $2 \mu \mathrm{s}$ & $0.29 \AA \times 0.29 \AA$ & 221 e $\AA^{-2}$ \\
\hline S15A,B,E,F & $1.49 \mathrm{pA}$ & $2 \mu \mathrm{s}$ & $0.29 \AA \times 0.29 \AA$ & 221 e $\AA^{-2}$ \\
\hline $\mathrm{S} 15 \mathrm{C}$ & $1.49 \mathrm{pA}$ & $2 \mu \mathrm{s}$ & $0.42 \AA \times 0.42 \AA$ & 105 e $\AA^{-2}$ \\
\hline S15D & $1.49 \mathrm{pA}$ & $2 \mu \mathrm{s}$ & $0.53 \AA \times 0.53 \AA$ & 66 e $\AA^{-2}$ \\
\hline
\end{tabular}




\section{Filtering}

Butterworth filtering adds a low- or high-pass filter across the Fourier transformation of the real-space image before reconstructing the image from the filtered FT, increasing the contrast of the image uniformly, as seen in Fig. S1B,E $(59,60)$. This approach makes it possible to retain information at grain boundaries, as seen on the left-hand side of Fig. S4B,E, but while it improves the overall contrast ratio dramatically, the noise is also amplified. All of the Butterworth-filtered images in this paper have been filtered using a $1^{\text {st }}$ order filter with a halfpoint of between 0.02 and 0.05 and a radius of 80 .

Bragg filtering imposes a strict periodic mask on the Fourier transformation, only maintaining the signal from a selected set of spots in the FT, as seen in Fig. S1C,F(61). This process removes all signal not included in the spots, giving the highest signal-to-noise ratio. This makes the atomic structure very visible, but also removes any features not included in the mask, leading, for example, to weakening of grain boundary information, as seen in Fig. S4C: the right-hand grain has excellent contrast, but the left-hand grain is blurry from the filtering. Choosing the appropriate filtering is, therefore, essential in order for reliable information to be obtainable. If information relating to the local atomic positions in the structure is desired, Bragg filtering should be applied, and where more macroscopic phenomena, such as grain boundaries, are studied, Butterworth filtering is appropriate.

The film area imaged and displayed in Fig. S1A-C contains both pristine and damaged perovskite as evidenced by the chequered pattern most clearly seen in the top right corner of the filtered images, as well as the additional periodicity observed in the Fourier transformations.

\section{LAADF multislice simulation}

The multislice simulations were performed using the MUSTEM software $(62,63)$ with a thickness of $30 \mathrm{~nm}$ and other parameters chosen to match the experimental LAADF acquisition. Aberrations were assumed to be negligible. The highly mobile $\mathrm{FA}^{+}$ion was modelled as a single particle, located at the notional carbon position with mean square displacements from the XRD literature(64). Multislice simulations are not able to quantitatively calculate the scattering intensity of the rotating $\mathrm{FA}^{+}$ions precisely and as a result, the intensity of the $\mathrm{FA}^{+}$ columns in Fig. S4D and Fig. S10 is underestimated but the relative locations are correct. The brightness and contrast of the image were adjusted to clearly illustrate the geometry of the material, which however makes the $\mathrm{Pb} / \mathrm{I}$ columns appear saturated

\section{Geometric phase analysis (GPA) strain map}

GPA strain mapping (Fig. S11) was done using the open-source Strain++ program written by Jon Peters $(65)$ and based on the algorithm in ref. (66). The g-vectors were chosen to correspond to the $200_{\mathrm{c}}$ and $020_{\mathrm{c}}$ spots in the Fourier transform and the resulting $\varepsilon_{\mathrm{xx}}$ strain map was overlaid on the corresponding Butterworth-filtered LAADF image. The strain profile in Fig. S11 B was extracted from the strain map using Digital Micrograph. 


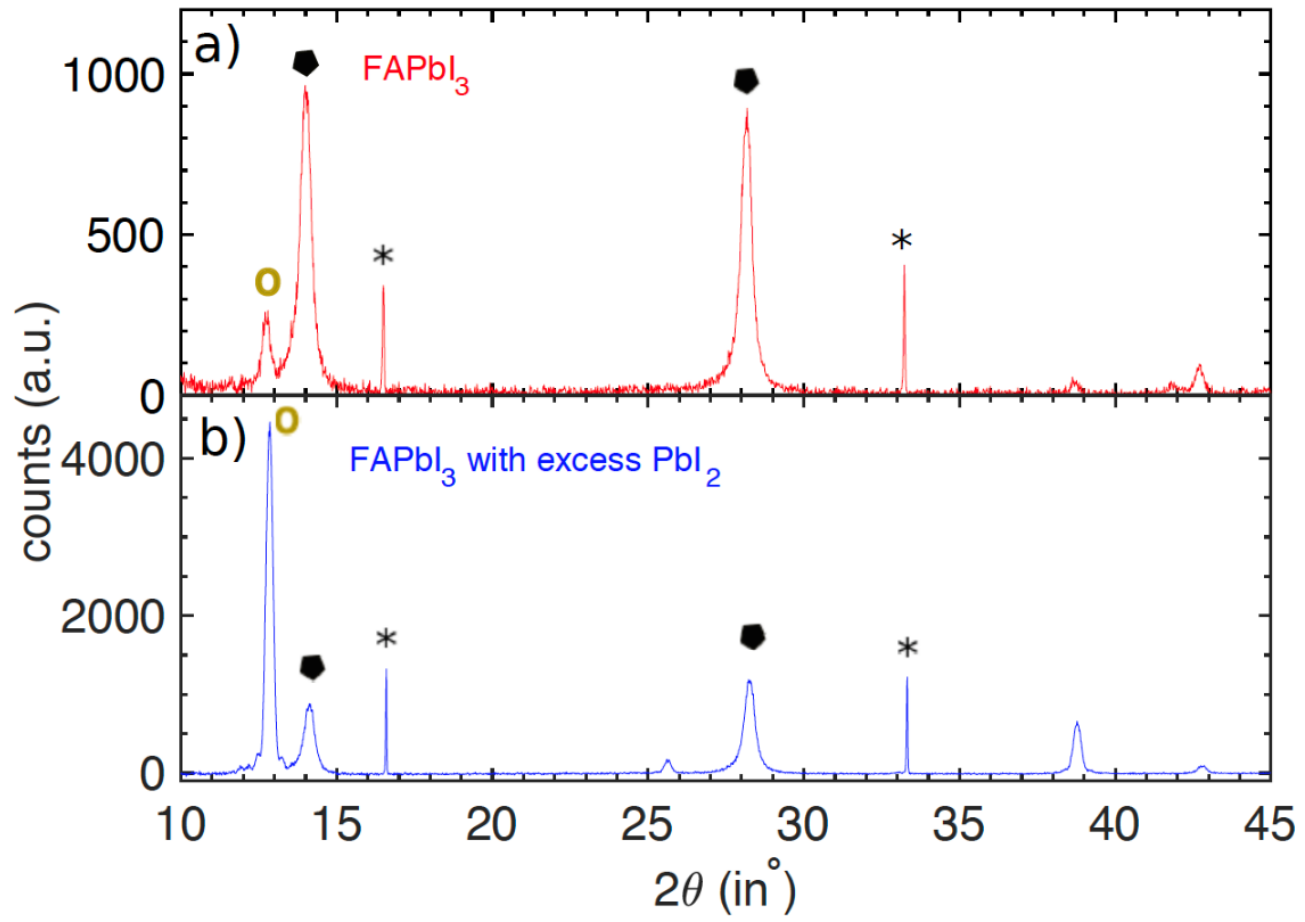

Fig. S1: X-ray diffraction patterns of $\mathbf{F A P b I}_{3}$ films measured with a Panalytical X'pert powder diffractometer for (a) near-stoichiometric $\mathrm{FAPbI}_{3}$ and (b) $\mathrm{FAPbI}_{3}$ deposited under $\mathrm{PbI}_{2}-$ rich conditions. Thin-films were deposited from dual-source vapour protocols analogous to those described in the Methods section, but on quartz substrates which allowed for signal to be collected from areas larger than those defined by the copper TEM grids, and facilitated angle calibration with respect to the known quartz diffraction peaks (marked by stars). Film thicknesses were the same as those of the TEM samples. The background was subtracted from the spectra. The characteristic perovskite diffraction peaks (marked by black pentagons) are clearly visible near $14^{\circ}$ and $28^{\circ}$. The near-stoichiometry $\mathrm{FAPbI}_{3}$ film only exhibits a small $\mathrm{PbI}_{2}$ diffraction peak (marked by beige circles) while the $\mathrm{PbI}_{2}$-rich $\mathrm{FAPbI}_{3}$ film fabricated with significant excess $\mathrm{PbI}_{2}$ exhibits a much stronger $\mathrm{PbI}_{2}$ peak. 

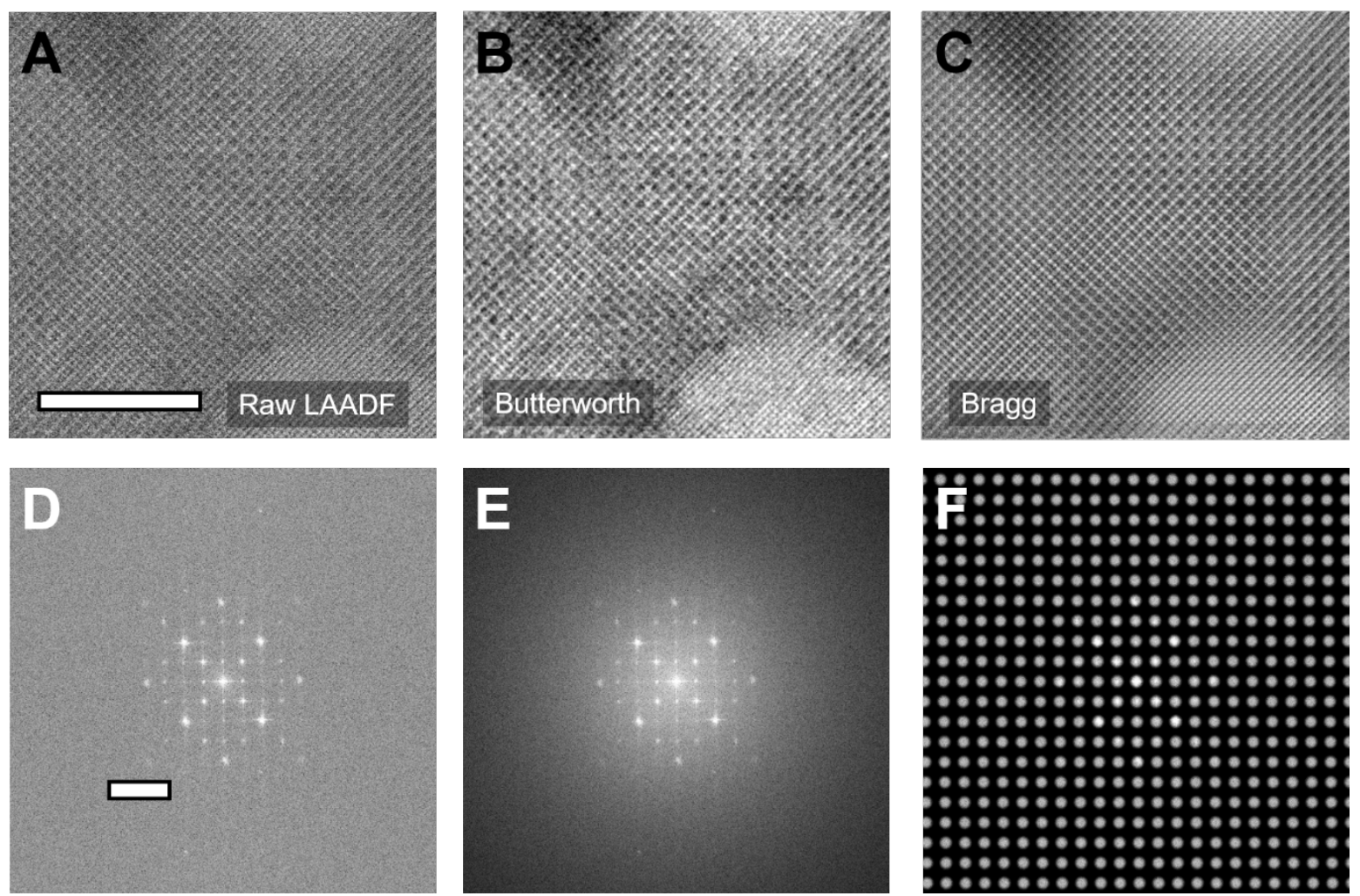

Fig. S2: Different filtering methods and their associated Fourier transformations. (A,D) show the raw LAADF image of an area of a $\mathrm{FAPbI}_{3}$ thin film and its associated Fourier transformation, respectively. (B,E) show the same area and Fourier transformation after a Butterworth low-pass filter has been applied. (C,F) show the same area and Fourier transformation after a Bragg filter has been applied. The real-space scale bar represents $10 \mathrm{~nm}$ and applies to (A-C). The Fourier space scale bar represents $4 \mathrm{~nm}^{-1}$ and applies to (D-F). 

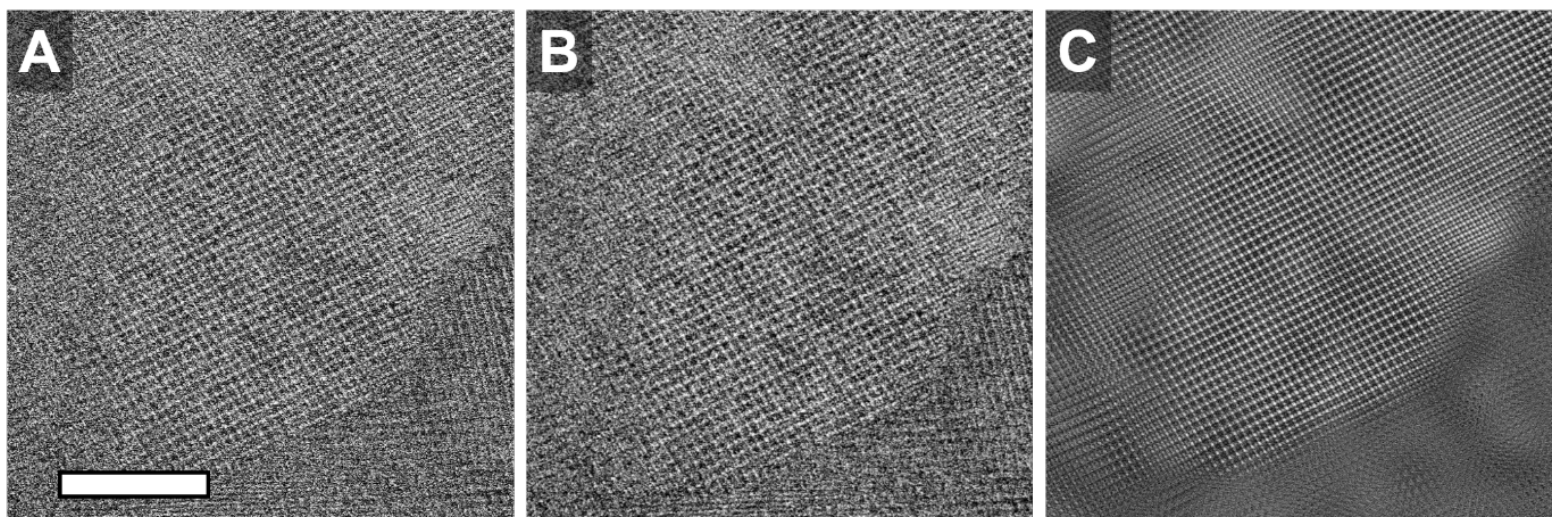

Fig. S3: Atomic-resolution HAADF micrographs of pristine $\mathbf{P b I}_{2}$-rich $\mathrm{FAPbI}_{3}$. (A) Raw data, (B) Butterworth filtered, (C) Bragg filtered. Images were obtained using a detector setting with 72.80 and $235.75 \mathrm{mrad}$ as the inner and outer collection angles, respectively. The scale bar represents $10 \mathrm{~nm}$. 

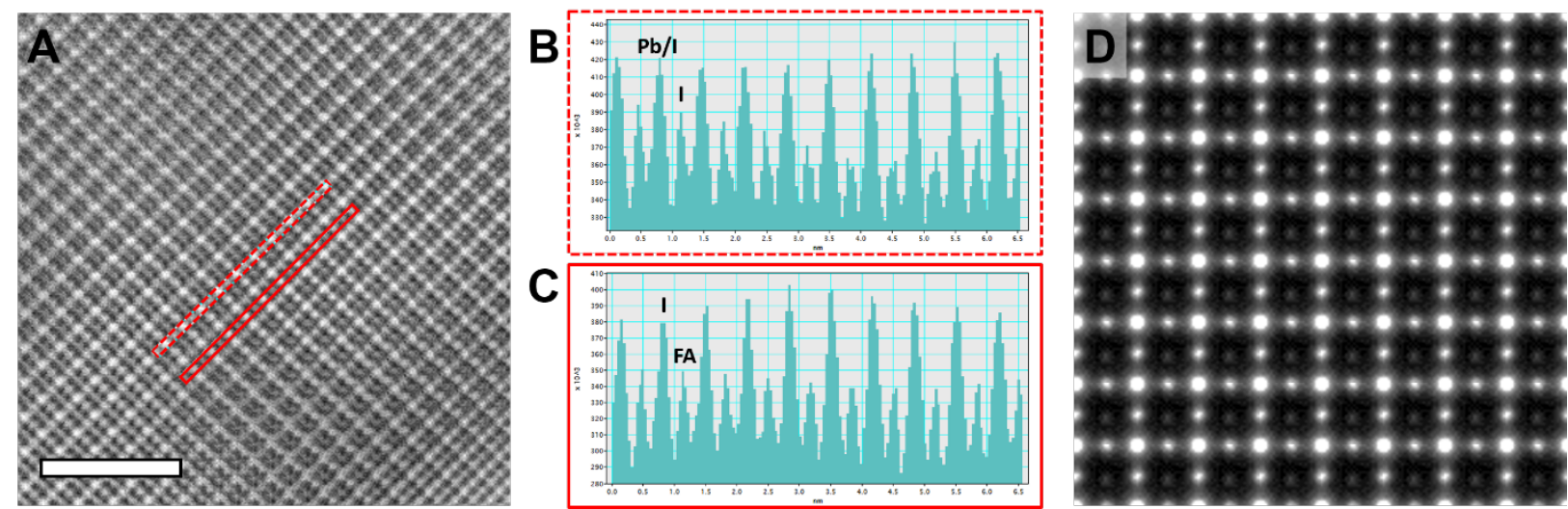

Fig. S4: Intensity line profiles across the $\mathrm{Pb} / \mathrm{I}$ and the I/FA lattice sites. (A) shows a Braggfiltered atomic resolution micrograph of a $\mathrm{FAPbI}_{3}$ thin film similar to that displayed in Fig. 1 of the main manuscript. (B,C) show the intensity line profiles obtained from the dashed and solid rectangles in (A), respectively. The scale bar in (A) represents $5 \mathrm{~nm}$. (D) Multislice simulation of the LAADF signal obtained from $\mathrm{FAPbI}_{3}$ when using parameters chosen to match the experimental LAADF acquisition. 

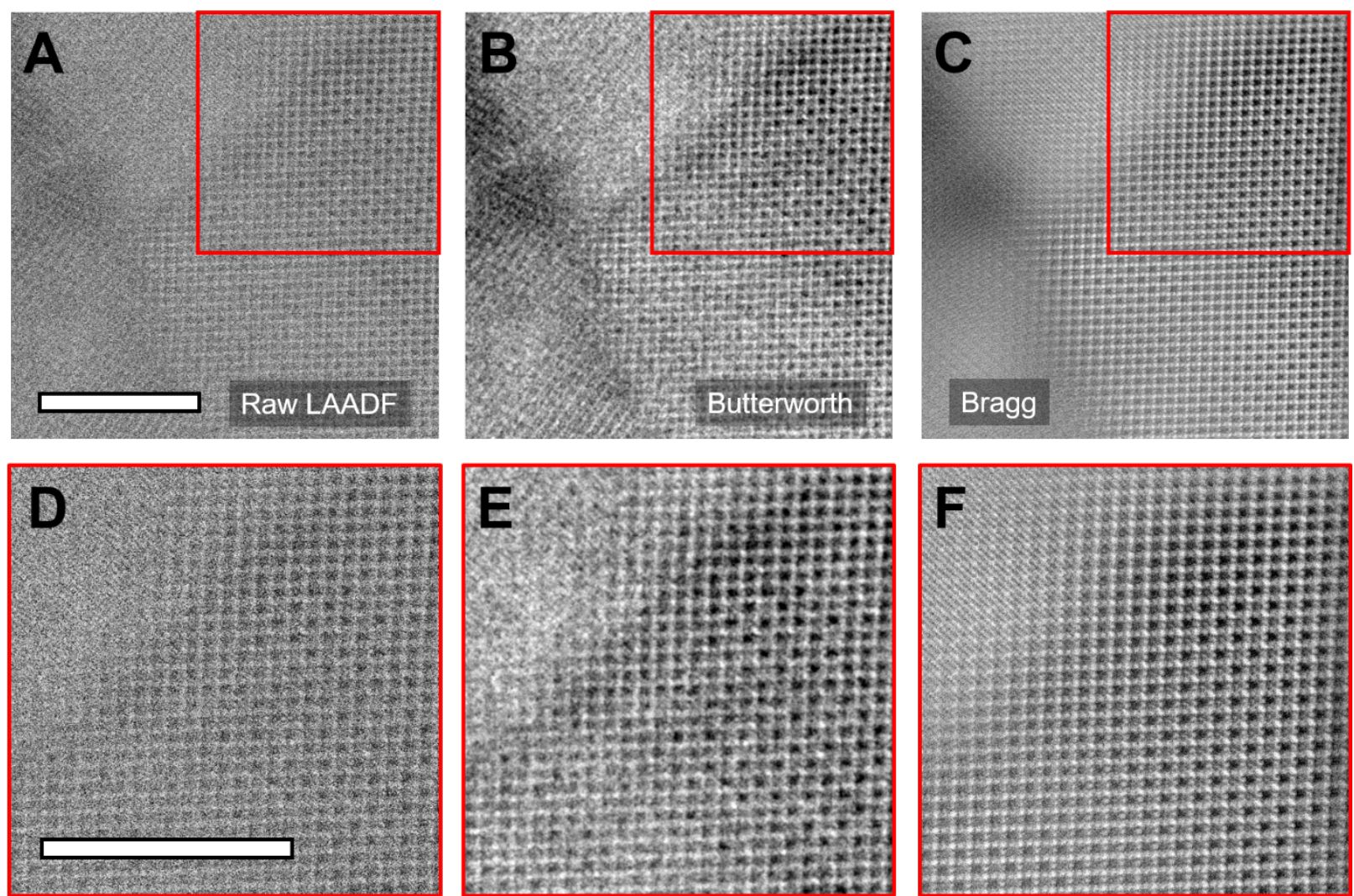

Fig. S5: Atomic-resolution LAADF micrographs of $\mathrm{FAPbI}_{3}$ thin films prepared with excess $\mathrm{PbI}_{2}$ showing a chequered intensity after mild radiation damage and seamless transitions between $\mathbf{P b I}_{2}$ and $\mathbf{F A P b I}_{3}$. (A-C) show real-space $\mathrm{LAADF}$ images of a $\mathrm{FAPbI}_{3}$ thin film with (A) no filter applied, (B) a Butterworth low-pass filter applied, and (C) a Bragg filter applied. (D-F) show the corresponding areas marked in the red squares in (A-C). The raw LAADF images are noisy because of the low number of electrons used in order to minimise beam damage. Applying a Butterworth filter dramatically improves the signal-to-noise ratio, making grain boundaries evident. A Bragg filter further improves the signal-to-noise ratio, but does not maintain information from the grain boundaries. Both filters make the $\mathrm{PbI}_{2}-\mathrm{FAPbI}_{3}$ intergrowth in the top of the images visible, and the presence of the $\mathrm{PbI}_{2}$ fringes in (F) confirms the close crystallographic relationship between the two phases. Both filters also make the chequered damage pattern in the right-hand grain very visible. The scale bars represent $10 \mathrm{~nm}$. 
Science Submitted Manuscript: Confidential

MAAAS

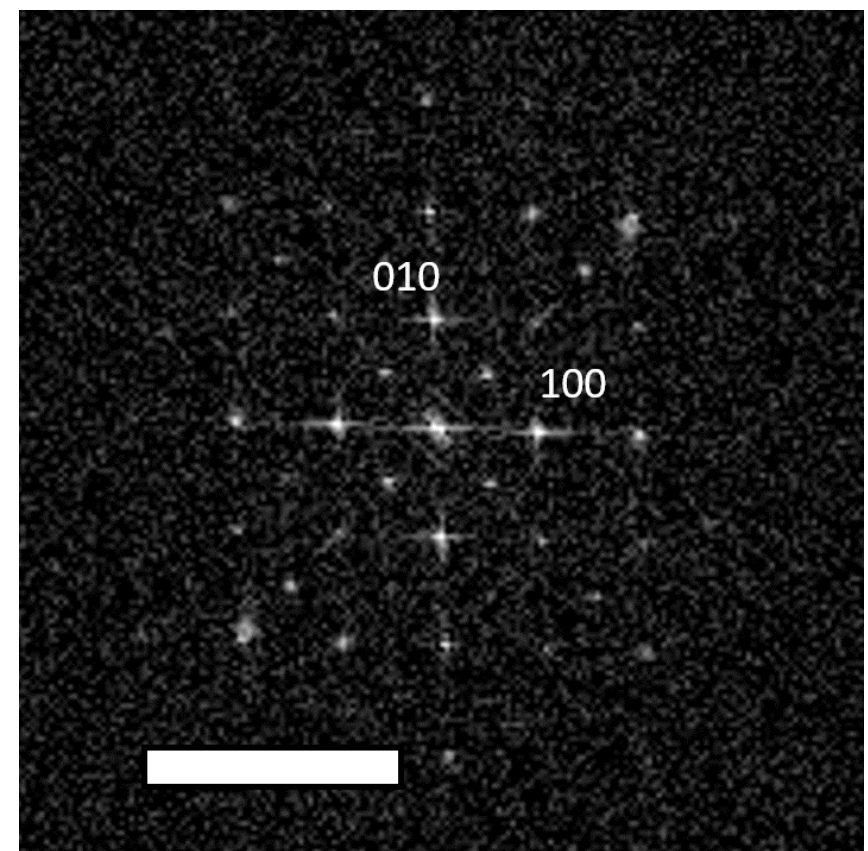

Fig. S6: Fourier transform of Fig. 3A. The scale bar represents $4 \mathrm{~nm}^{-1}$. 

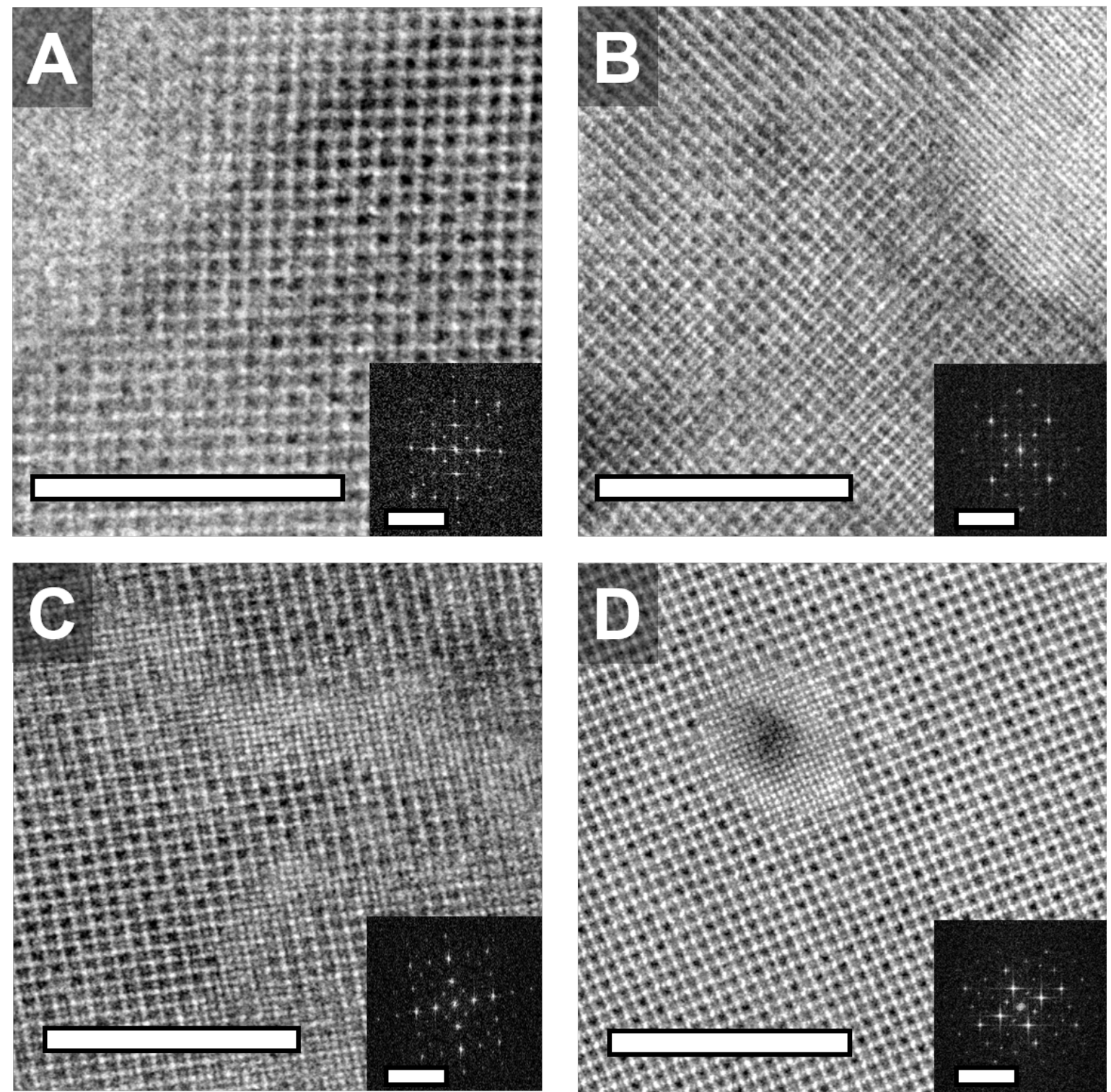

Fig. S7: Atomic-resolution LAADF micrographs of $\mathrm{FAPbI}_{3}$ thin films showing undistorted transitions between $\mathrm{PbI}_{2}$ and $\mathrm{FAPbI}_{3}$, as well as a chequered intensity after mild radiation damage without colour overlay. All micrographs have been filtered using Butterworth filtering. (A-C) were prepared with significant excess $\mathrm{PbI}_{2}$ and (D) is nearstoichiometric. The inserts show the Fourier transform of the real-space images, illustrating the epitaxial nature of the interfaces. The scale bars represent $10 \mathrm{~nm}$ in the real-space images and $4 \mathrm{~nm}^{-1}$ in the inserted Fourier transforms. 

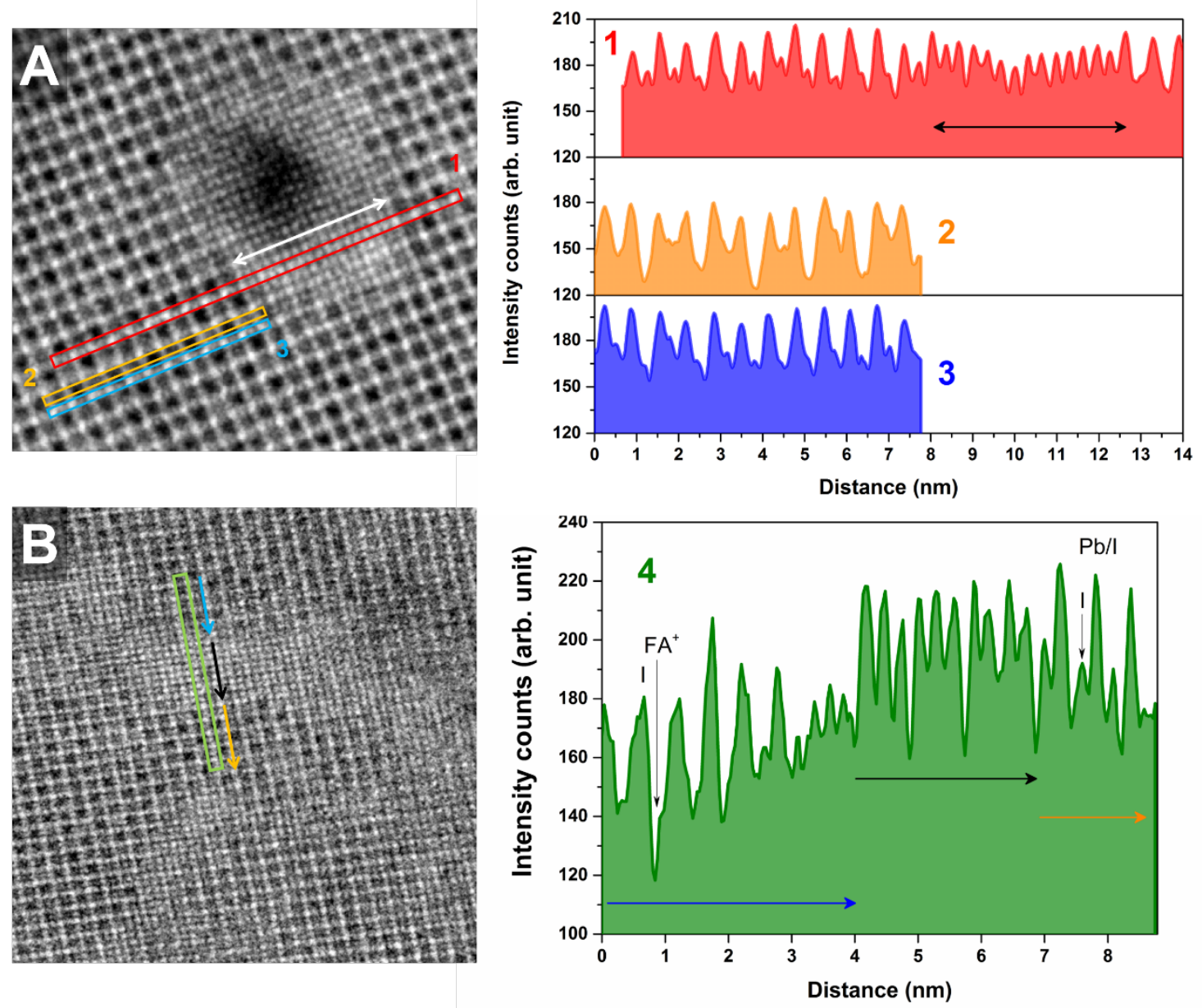

Fig. S8: Intensity line profiles of different atomic columns in $\mathrm{FAPbI}_{3}$. (A) Line profile 1 (red) shows the intensity variation across $\mathrm{Pb} / \mathrm{I}$ and I columns of damaged $\mathrm{FAPbI}_{3}$ and the columns in the associated $\mathrm{PbI}_{2}$. The intensity of the signal decreases within the $\mathrm{PbI}_{2}$ region (marked by the black and white arrows), indicating a local loss of material. Line profile 2 (yellow) shows the ordering of the $\mathrm{FA}^{+}$columns with every other column being either partially or fully empty, indicating a columnar redistribution of the $\mathrm{FA}^{+}$after beam damage. Line profile 3 (blue) shows minimal evidence of ordered loss of $\mathrm{I}^{-}$, suggesting that the $\left[\mathrm{PbI}_{6}\right]$ octahedral units can remain intact in the perovskite lattice despite the loss of $\mathrm{FA}^{+}$. (B) Line profile 4 (green) shows the intensity variation across the perovskite/ $\mathrm{PbI}_{2}$ intergrowth causing half a unit cell shit, displayed in Fig. 3C. The LAADF signal intensity of the $\mathrm{FA}^{+} / \mathrm{I}$ columns (blue arrow) is lower than that of the I/Pb:I columns (orange arrow), illustrating the shift of half a unit cell across the $\mathrm{PbI}_{2}$ (black arrow). The composition of the film imaged in (A) is near-stoichiometric, and in (B) as prepared with significant excess $\mathrm{PbI}_{2}$. 

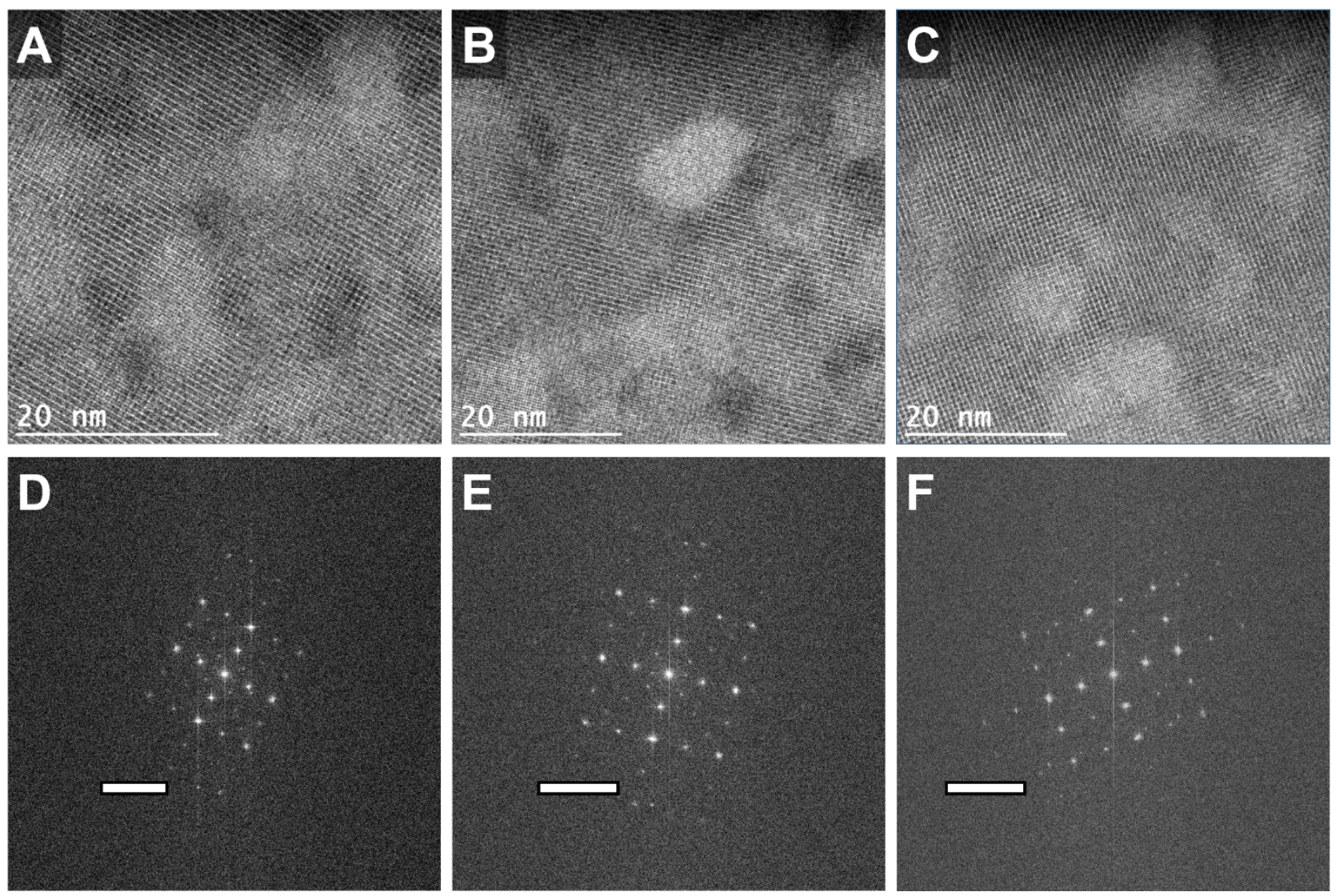

Figure S9: $\mathbf{P b I}_{2}$ incorporation into $\mathrm{FAPbI}_{3}$ prepared with significant excess $\mathbf{P b I}_{2}$. (A-C)

Representative Butterworth-filtered LAADF micrographs showing coherent incorporation of $\mathrm{PbI}_{2}$ clusters into the surrounding perovskite lattice. (D-F) Fourier transforms of the micrographs taken before Butterworth filtering, showing only a single pattern in each transform, indicating that the $\mathrm{PbI}_{2}$ is incorporated epitaxially into the perovskite lattice in all directions. The scale bars in (D-F) represent $4 \mathrm{~nm}^{-1}$. 
$\mathrm{FAPbI}_{3} / \mathrm{PbI}_{2}$ interface

The structure of $\mathrm{PbI}_{2}$ comprises $\left[\mathrm{PbI}_{6}\right]$ octahedra that form $2 \mathrm{D}$ layers which interact through van der Waals forces with neighbouring layers. The I-I distance across the $\mathrm{PbI}_{2}$ octahedra $(0.64 \mathrm{~nm}(42))$ is very close to the corresponding distance in the similar octahedra in $\mathrm{FAPbI}_{3}$ $(0.63 \mathrm{~nm})$. The structure of the $2 \mathrm{H}$ polytype viewed along [110] is shown in the top of Fig. S10.

The weak van der Waals interaction between layers leads to extensive polytypism in $\mathrm{PbI}_{2}$ corresponding to various shifts between the layers. The bottom of Fig. S10 shows how a small shift in the registration of the layers and a slight compression of the gap between the layers can lead to a structure that is epitaxial and coherent with the cubic perovskite structure with the $\left[\mathrm{PbI}_{6}\right]$ octahedra being continuous across the two structures.

This interpretation of the interface structure also explains the relative intensities of the atomic columns in the images in Fig. 3 and Fig. S8. The most intense columns are those in the perovskite with the atomic sequence I-Pb-I-Pb-I-Pb-I. The next most intense columns are those in the $\mathrm{PbI}_{2}$ phase that follow the sequence I-Pb-I-_-I-Pb-I where the underscore is the gap between the layers. They have consistent intensity in both the $2 \mathrm{H}$ structure and the distorted epitaxial structure in Fig 2 consistent with our model. The I-_-I-_-I columns in the perovskite follow in the weakening intensity sequence with finally the FA columns being the weakest. 


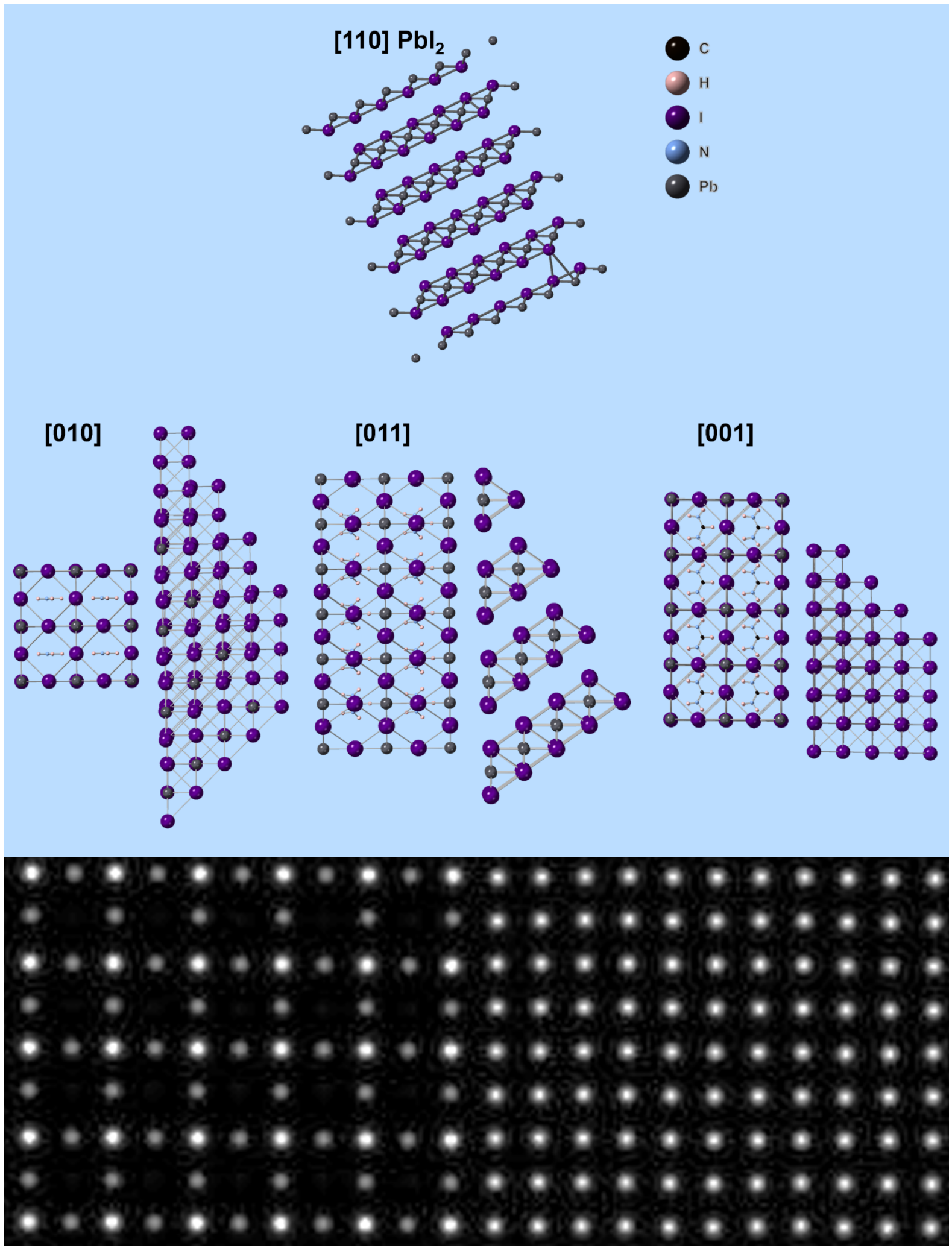

Fig. S10: Stick and ball model of the interface between $\mathrm{FAPbI}_{3}$ and slightly modified $2 \mathrm{H}$ $\mathbf{P b I}_{2}$ as well as LAADF signal intensity simulation of the interface. Top shows [100]oriented $2 \mathrm{H} \mathrm{PbI}_{2}$. Middle shows the interface between $\mathrm{FAPbI}_{3}$ and $\mathrm{PbI}_{2}$. The interface is shown in three different orientations, with the orientation index corresponding to the $\mathrm{FAPbI}_{3}$ orientation. The perovskite structure is displayed on the left and the $\mathrm{PbI}_{2}$ on the right of each interface. Bottom shows the simulated LAADF signal intensity across the interface along the perovskite $<100>$ axis, with the perovskite on the left and $\mathrm{PbI}_{2}$ on the right. The low intensity 
of the $\mathrm{FA}^{+}$columns caused by the multislice simulations being unable to quantitatively calculate the scattering intensity of the rotating $\mathrm{FA}^{+}$ions precisely.
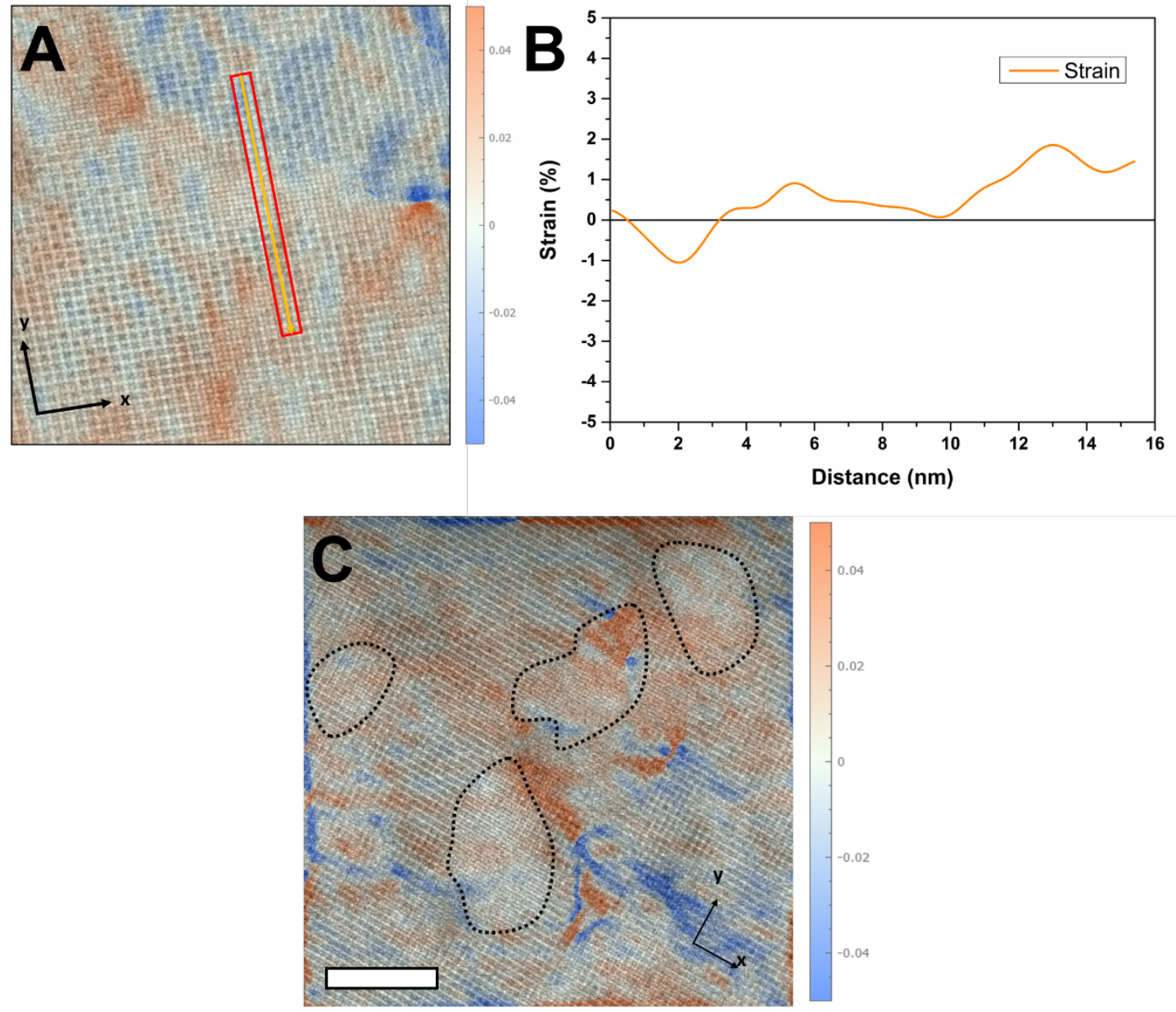

Fig. S11: Crystallographic strain across a $\mathbf{P b I}_{2} / \mathbf{F A P b I}_{3}$ interface. (A) Geometric phase analysis heat map of the $\varepsilon_{x x}$ strain showing virtually no strain associated with the interface. (B) Line profile of the strain within the red rectangle along the orange arrow. The strain across the interface varies from $-1 \%$ to $2 \%$. (C) Geometric strain analysis heat map of the $\varepsilon_{y y}$ strain map in the pristine $\mathrm{PbI}_{2}$-rich $\mathrm{FAPbI}_{3}$ film displayed in Fig. 9A, showing some atomic strain throughout the film. Some $\mathrm{PbI}_{2}$ inclusions are highlighted by the dotted lines, showing that there is no inherently higher strain associated with the $\mathrm{PbI}_{2} / \mathrm{FAPbI}_{3}$ interface than within the rest of the film. The scale bar represents $10 \mathrm{~nm}$. 

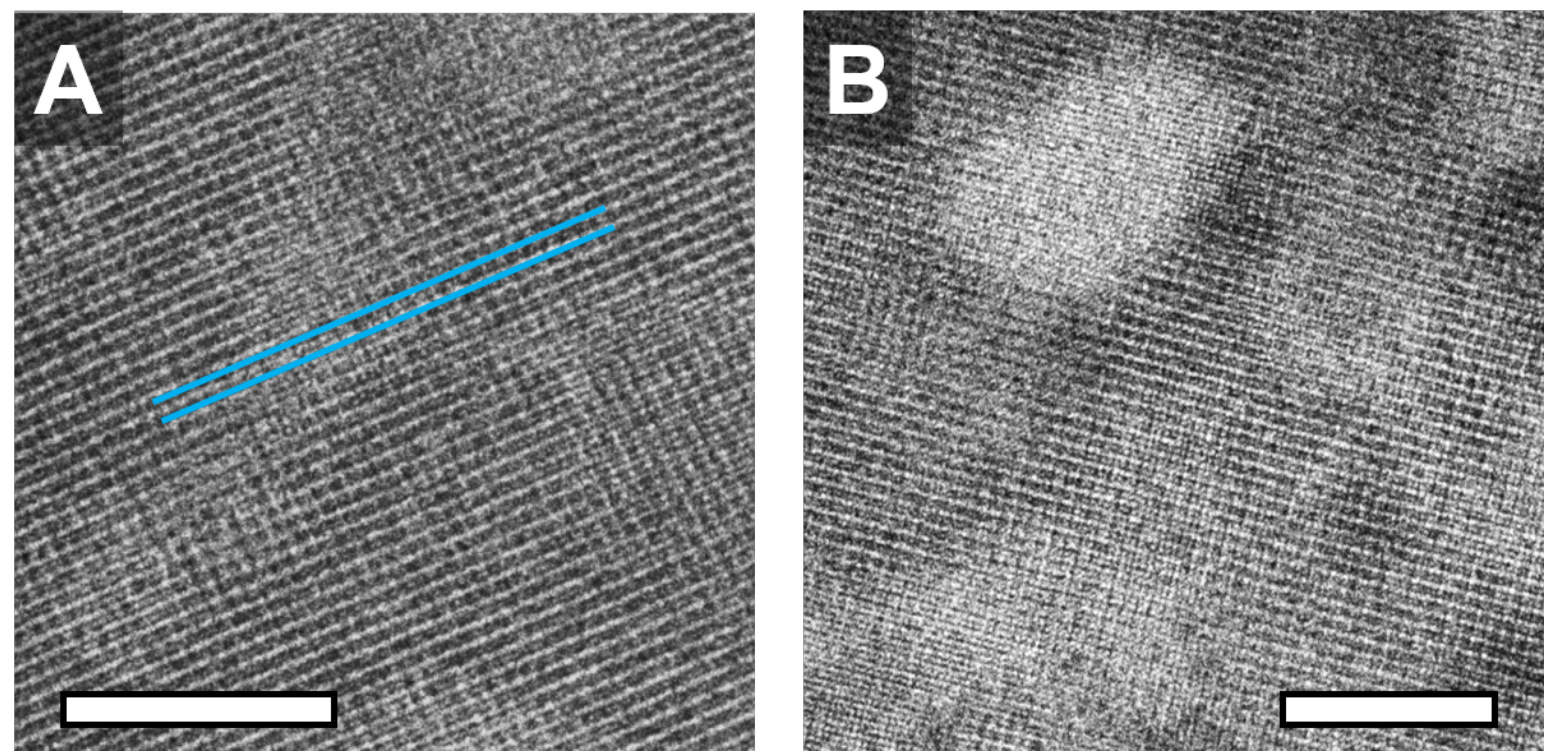

Fig. S12: Atomic-resolution LAADF micrographs of pristine $\mathrm{PbI}_{2}$-rich $\mathrm{FAPbI}_{3}$ showing (A) shift of half a perovskite unit cell in pristine $\mathrm{FAPbI}_{3}$ and (B) $\mathrm{PbI}_{2}$ intergrowth with the pristine perovskite lattice. The scale bars represent $10 \mathrm{~nm}$. 
МIAAAS

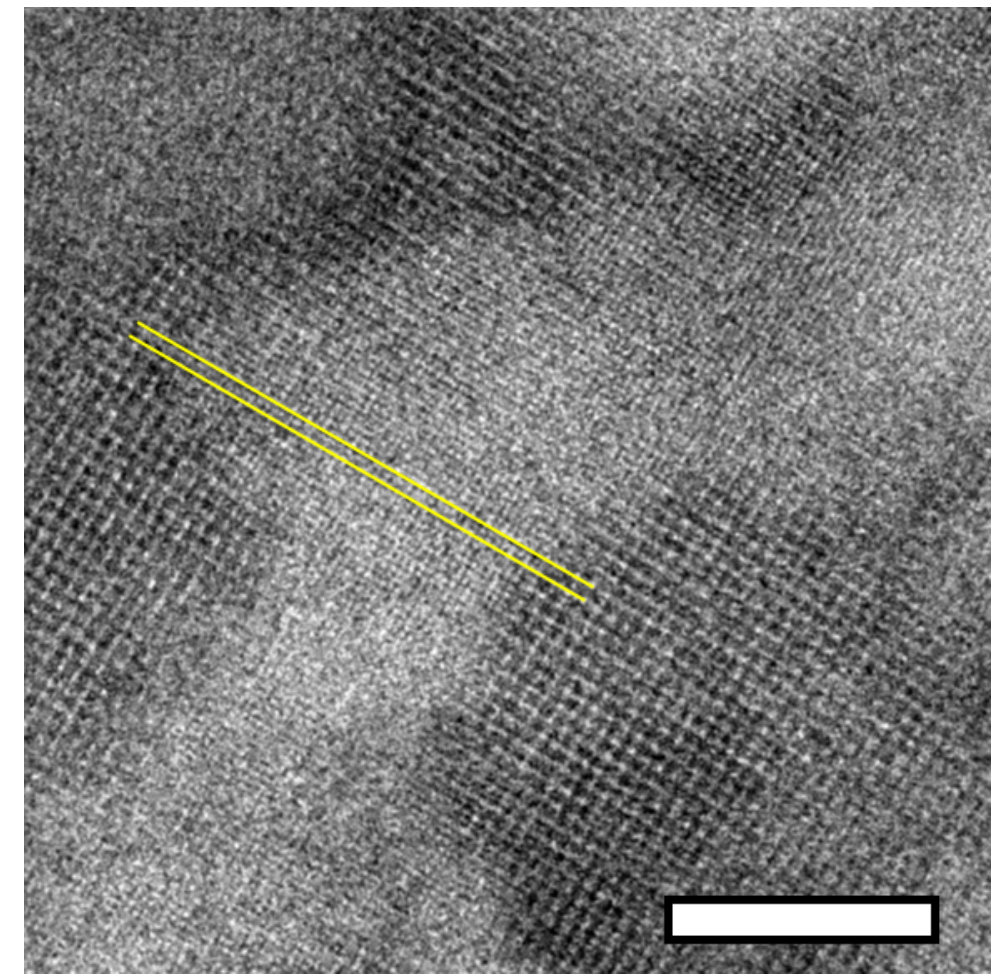

Fig. S13: Atomic-resolution LAADF micrograph of $\mathbf{P b I}_{2}$-rich $\mathrm{MAPbI}_{3}$ showing both the undistorted perovskite-to- $\mathrm{PbI}_{2}$ transitions and the shift of half a unit cell across a $\mathrm{PbI}_{2}$ region (parallel yellow lines). The scale bar represents $10 \mathrm{~nm}$. 

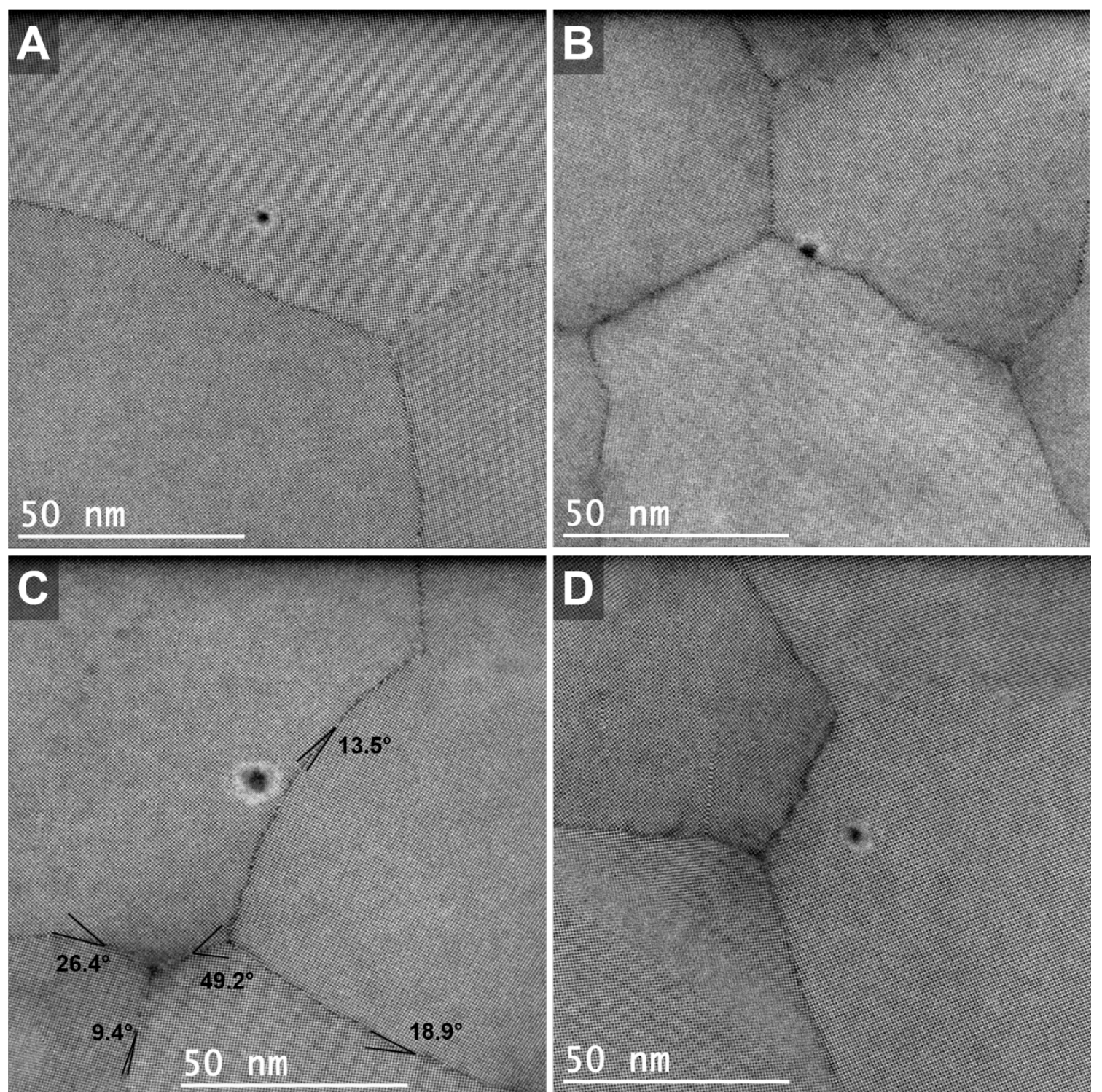

Fig. S14: Representative lower-magnification Butterworth-filtered atomic-resolution micrographs of $\mathbf{F A P b I}_{3}$ grains as seen in a $30-\mathrm{nm}$ thick film prepared near the correct stoichiometry. The round dark areas are regions damaged by the parked electron beam before image acquisition. Fig. 4A in the main manuscript is a magnified excerpt of (A), Fig. 4B is a magnified excerpt of (C), and Fig. 3D and Fig. 4E are magnified excerpts of (D). A wide range of grain boundary angles are evident in $(\mathrm{C})$, illustrating the highly conformal nature of $\mathrm{FAPbI}_{3}$. 

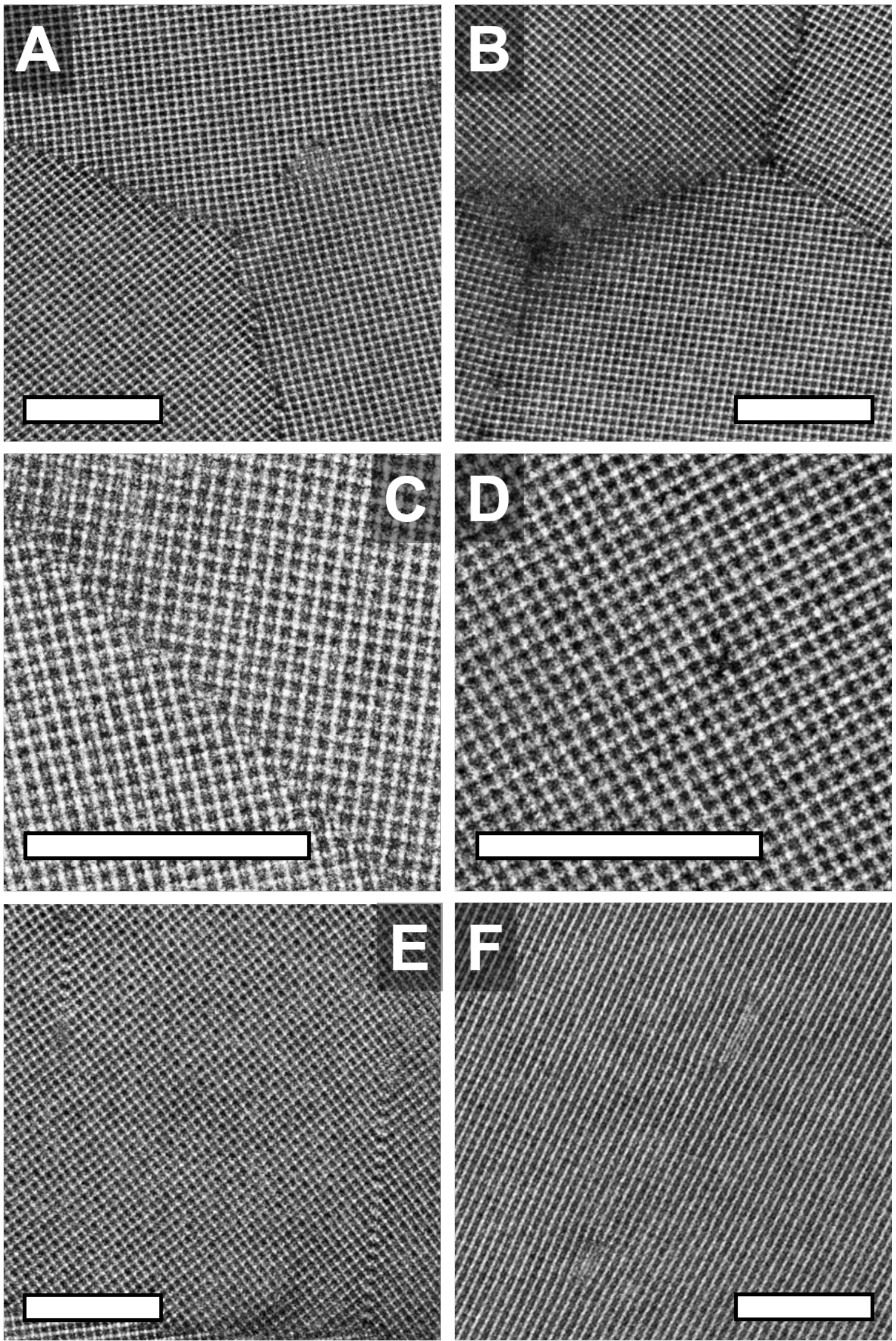

Fig. S15: Butterworth-filtered atomic-resolution micrographs of representative $\mathrm{FAPbI}_{3}$ grain boundaries and crystal defects as seen in a 30-nm thick film prepared near the correct stoichiometry, shown without colour overlay. The scale bars represent $10 \mathrm{~nm}$. 

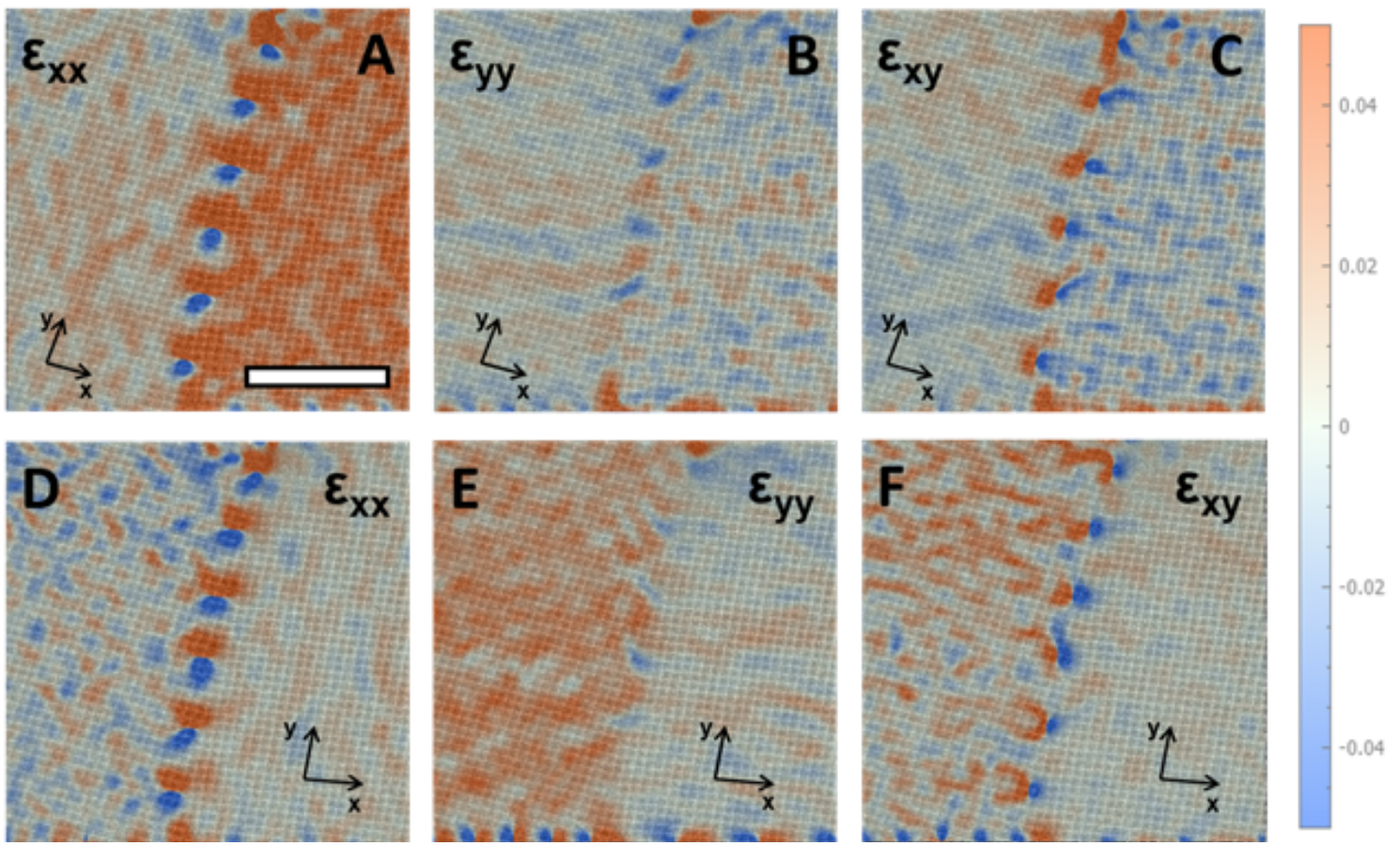

Fig. S16: GPA strain maps near the low-angle boundary between two near-stoichiometric FAPbI3 grains displayed in the bottom left of Fig. 4B. (A-C) show the $x x$, yy, and xy strain in the left-hand grain, and (D-F) show the $\mathrm{xx}$, yy, and xy strain in the right-hand grain, respectively. A periodic pattern of $\mathrm{xx}$ and $\mathrm{xy}$ strain with alternating sign is evident and arises from the periodic array of edge dislocations forming the grain boundary, whereas the yy strain (lying close to perpendicular to the dislocation burgers vectors) is much lower at the boundary. All significant strain is localised to within one or two unit cells from the boundary, showing that the boundary does not induce long-range strain in the grains themselves. The inserted coordinate axes show the respective defined lattice directions. The periodic strain patterns at the bottom of each image are due to edge effects and are not physical. The scale bar represents $10 \mathrm{~nm}$ and applies to all the images. 


\section{References}

1. H.-S. Kim et al., Lead iodide perovskite sensitized all-solid-state submicron thin film mesoscopic solar cell with efficiency exceeding 9\%. Scientific reports 2, 591 (2012).

2. M. Liu, M. B. Johnston, H. J. Snaith, Efficient planar heterojunction perovskite solar cells by vapour deposition. Nature 501, 395 (2013).

3. H. Zhou et al., Interface engineering of highly efficient perovskite solar cells. Science 345, 542-546 (2014).

4. N. J. Jeon et al., Solvent engineering for high-performance inorganic-organic hybrid perovskite solar cells. Nat Mater 13, 897-903 (2014).

5. J.-P. Correa-Baena et al., The rapid evolution of highly efficient perovskite solar cells. Energy \& Environmental Science 10, 710-727 (2017).

6. M. Saliba et al., Incorporation of rubidium cations into perovskite solar cells improves photovoltaic performance. Science 354, 206-209 (2016).

7. D. P. McMeekin et al., A mixed-cation lead mixed-halide perovskite absorber for tandem solar cells. Science 351, 151-155 (2016).

8. D. W. DeQuilettes et al., Photo-induced halide redistribution in organic-inorganic perovskite films. Nature communications 7, 1-9 (2016).

9. C. C. Stoumpos, C. D. Malliakas, M. G. Kanatzidis, Semiconducting tin and lead iodide perovskites with organic cations: phase transitions, high mobilities, and near-infrared photoluminescent properties. Inorganic chemistry 52, 9019-9038 (2013).

10. T. Baikie et al., A combined single crystal neutron/X-ray diffraction and solid-state nuclear magnetic resonance study of the hybrid perovskites $\mathrm{CH} 3 \mathrm{NH} 3 \mathrm{PbX} 3$ (X= I, $\mathrm{Br}$ and $\mathrm{Cl}$ ). Journal of Materials Chemistry A 3, 9298-9307 (2015).

11. M. U. Rothmann, W. Li, J. Etheridge, Y. B. Cheng, Microstructural Characterisations of Perovskite Solar Cells-From Grains to Interfaces: Techniques, Features, and Challenges. Advanced Energy Materials 7, (2017).

12. K. Domanski, E. A. Alharbi, A. Hagfeldt, M. Grätzel, W. Tress, Systematic investigation of the impact of operation conditions on the degradation behaviour of perovskite solar cells. Nature Energy 3, 61-67 (2018).

13. L. Hong et al., Improvement and Regeneration of Perovskite Solar Cells via Methylamine Gas Post-Treatment. Advanced Functional Materials 27, 1703060 (2017).

14. D. H. Cao et al., Remnant PbI2, an unforeseen necessity in high-efficiency hybrid perovskite-based solar cells? Apl Materials 2, 091101 (2014).

15. H.-Y. Hsu et al., Optimization of $\mathrm{PbI} 2 / \mathrm{MAPbI} 3$ perovskite composites by scanning electrochemical microscopy. The Journal of Physical Chemistry C 120, 19890-19895 (2016).

16. Y. C. Kim et al., Beneficial effects of PbI2 incorporated in organo-lead halide perovskite solar cells. Advanced Energy Materials 6, 1502104 (2016).

17. J.-W. Lee et al., The role of grain boundaries in perovskite solar cells. Materials Today Energy 7, 149-160 (2018).

18. R. Long, J. Liu, O. V. Prezhdo, Unravelling the effects of grain boundary and chemical doping on electron-hole recombination in $\mathrm{CH} 3 \mathrm{NH} 3 \mathrm{PbI} 3$ perovskite by time-domain atomistic simulation. Journal of the American Chemical Society 138, 3884-3890 (2016).

19. T. Niu et al., Stable High-Performance Perovskite Solar Cells via Grain Boundary Passivation. Advanced Materials 30, 1706576 (2018).

20. S. Jariwala et al., Local crystal misorientation influences non-radiative recombination in halide perovskites. Joule 3, 3048-3060 (2019). 
21. C. L. Davies et al., Impact of the organic cation on the optoelectronic properties of formamidinium lead triiodide. The journal of physical chemistry letters 9, 4502-4511 (2018).

22. S. K. Yadavalli et al., Mechanisms of Exceptional Grain Growth and Stability in Formamidinium Lead Triiodide Thin Films for Perovskite Solar Cells. Acta Materialia, (2020).

23. A. Knight, L. M. Herz, Preventing phase segregation in mixed-halide perovskites: a perspective. Energy \& Environmental Science, (2020).

24. O. Breitenstein, J. Rakotoniaina, M. H. Al Rifai, M. Werner, Shunt types in crystalline silicon solar cells. Progress in Photovoltaics: Research and Applications 12, 529-538 (2004).

25. A. Stoffers et al., Complex Nanotwin Substructure of an Asymmetric $\Sigma 9$ Tilt Grain Boundary in a Silicon Polycrystal. Physical review letters 115, 235502 (2015).

26. Y. Yu et al., Atomic resolution imaging of halide perovskites. Nano letters 16, 75307535 (2016).

27. D. Zhang et al., Atomic-resolution transmission electron microscopy of electron beamsensitive crystalline materials. Science 359, 675-679 (2018).

28. Y. Li et al., Unravelling degradation mechanisms and atomic structure of organicinorganic halide perovskites by cryo-EM. Joule 3, 2854-2866 (2019).

29. M. U. Rothmann et al., Structural and chemical changes to $\mathrm{CH} 3 \mathrm{NH} 3 \mathrm{PbI} 3$ induced by electron and gallium ion beams. Advanced Materials 30, 1800629 (2018).

30. S. Chen et al., Atomic scale insights into structure instability and decomposition pathway of methylammonium lead iodide perovskite. Nature communications 9, 1-8 (2018).

31. R. Hooley, A. Brown, A. Kulak, F. Meldrum, R. Brydson, in Journal of Physics: Conference Series. (IOP Publishing, 2017), vol. 902, pp. 012005.

32. K. B. Lohmann et al., Control over crystal size in vapor deposited metal-halide perovskite films. ACS Energy Letters 5, 710-717 (2020).

33. J. Borchert et al., Large-area, highly uniform evaporated formamidinium lead triiodide thin films for solar cells. ACS Energy Letters 2, 2799-2804 (2017).

34. J. Gonnissen et al., Optimal experimental design for the detection of light atoms from high-resolution scanning transmission electron microscopy images. Applied Physics Letters 105, 063116 (2014).

35. L. Oesinghaus et al., Toward tailored film morphologies: the origin of crystal orientation in hybrid perovskite thin films. Advanced Materials Interfaces 3, 1600403 (2016).

36. H. J. Jung et al., Stability of halide perovskite solar cell devices: in situ observation of oxygen diffusion under biasing. Advanced Materials 30, 1802769 (2018).

37. T. W. Kim et al., Self-Organized Superlattice and Phase Coexistence inside Thin Film Organometal Halide Perovskite. Advanced Materials 30, 1705230 (2018).

38. Z. Chen, Z.-G. Gu, W.-Q. Fu, F. Wang, J. Zhang, A confined fabrication of perovskite quantum dots in oriented MOF thin film. ACS applied materials \& interfaces 8, 2873728742 (2016).

39. M. Xiao et al., A Fast Deposition-Crystallization Procedure for Highly Efficient Lead Iodide Perovskite Thin-Film Solar Cells. Angewandte Chemie 126, 10056-10061 (2014).

40. Y. Zhou, H. Sternlicht, N. P. Padture, Transmission electron microscopy of halide perovskite materials and devices. Joule 3, 641-661 (2019).

41. J. Ran et al., Electron-Beam-Related Studies of Halide Perovskites: Challenges and Opportunities. Advanced Energy Materials, 1903191 (2020). 
42. R. Egerton, Radiation damage to organic and inorganic specimens in the TEM. Micron 119, $72-87$ (2019).

43. R. F. Egerton, P. Li, M. Malac, Radiation damage in the TEM and SEM. Micron 35, 399-409 (2004).

44. J. Ran et al., Electron-Beam-Related Studies of Halide Perovskites: Challenges and Opportunities. Advanced Energy Materials n/a, 1903191.

45. P. A. Beckmann, A review of polytypism in lead iodide. Crystal Research and Technology 45, 455-460 (2010).

46. H.-S. Duan et al., The identification and characterization of defect states in hybrid organic-inorganic perovskite photovoltaics. Physical chemistry chemical physics 17, 112-116 (2015).

47. X. Wen et al., Defect trapping states and charge carrier recombination in organicinorganic halide perovskites. Journal of Materials Chemistry C 4, 793-800 (2016).

48. D. W. de Quilettes et al., Impact of microstructure on local carrier lifetime in perovskite solar cells. Science 348, 683-686 (2015).

49. Q. Chen et al., Controllable self-induced passivation of hybrid lead iodide perovskites toward high performance solar cells. Nano letters 14, 4158-4163 (2014).

50. Y. Shao, Z. Xiao, C. Bi, Y. Yuan, J. Huang, Origin and elimination of photocurrent hysteresis by fullerene passivation in $\mathrm{CH} 3 \mathrm{NH} 3 \mathrm{PbI} 3$ planar heterojunction solar cells. Nature communications 5, 1-7 (2014).

51. A. F. Castro-Méndez, J. Hidalgo, J. P. Correa-Baena, The role of grain boundaries in perovskite solar cells. Advanced Energy Materials 9, 1901489 (2019).

52. J. M. Ball, A. Petrozza, Defects in perovskite-halides and their effects in solar cells. Nature Energy 1, 1-13 (2016).

53. A. Merdasa et al., Super-resolution luminescence microspectroscopy reveals the mechanism of photoinduced degradation in $\mathrm{CH} 3 \mathrm{NH} 3 \mathrm{PbI} 3$ perovskite nanocrystals. The Journal of Physical Chemistry C 120, $10711-10719$ (2016).

54. C. Xiao et al., Mechanisms of Electron-Beam-Induced Damage in Perovskite Thin Films Revealed by Cathodoluminescence Spectroscopy. The Journal of Physical Chemistry C 119, 26904-26911 (2015).

55. J. B. Patel et al., Photocurrent spectroscopy of perovskite solar cells over a wide temperature range from 15 to $350 \mathrm{~K}$. The journal of physical chemistry letters $\mathbf{9}, 263-$ 268 (2018).

56. J. D. Zook, Effects of grain boundaries in polycrystalline solar cells. Applied Physics Letters 37, 223-226 (1980).

57. F. Greuter, G. Blatter, Electrical properties of grain boundaries in polycrystalline compound semiconductors. Semiconductor Science and Technology 5, 111 (1990).

58. J.-W. Lee et al., Formamidinium and Cesium Hybridization for Photo- and MoistureStable Perovskite Solar Cell. Advanced Energy Materials 5, 1501310-n/a (2015).

59. L. Kimerling, H. Leamy, J. Patel, The electrical properties of stacking faults and precipitates in heat-treated dislocation-free Czochralski silicon. Applied Physics Letters 30, 217-219 (1977).

60. T. W. Kim et al., Self-Organized Superlattice and Phase Coexistence inside Thin Film Organometal Halide Perovskite. Advanced Materials, 1705230-n/a.

61. J. Borchert et al., Impurity Tracking Enables Enhanced Control and Reproducibility of Hybrid Perovskite Vapor Deposition. ACS applied materials \& interfaces 11, 2885128857 (2019). 